\title{
Destabilization of nucleophosmin mRNA by the HuR/KSRP complex is required for muscle fibre formation
}

Anne Cammas ${ }^{1,2}$, Brenda Janice Sanchez ${ }^{1}$, Xian Jin Lian¹, Virginie Dormoy-Raclet ${ }^{1}$, Kate van der Giessen ${ }^{1}$, Isabel López de Silanes ${ }^{3}$, Jennifer Ma ${ }^{1}$, Carol Wilusz ${ }^{4}$, John Richardson ${ }^{5}$, Myriam Gorospe ${ }^{6}$, Stefania Millevoi ${ }^{2}$, Matteo Giovarelli ${ }^{7}$, Roberto Gherzi ${ }^{7}$, Sergio Di Marco ${ }^{1} \&$ Imed-Eddine Gallouzi ${ }^{1}$

HuR promotes myogenesis by stabilizing the MyoD, myogenin and p21 mRNAs during the fusion of muscle cells to form myotubes. Here we show that HuR, via a novel mRNA destabilizing activity, promotes the early steps of myogenesis by reducing the expression of the cell cycle promoter nucleophosmin (NPM). Depletion of HuR stabilizes the NPM mRNA, increases NPM protein levels and inhibits myogenesis, while its overexpression elicits the opposite effects. NPM mRNA destabilization involves the association of HuR with the decay factor KSRP as well as the ribonuclease PARN and the exosome. The $\mathrm{C}$ terminus of HuR mediates the formation of the HuR-KSRP complex and is sufficient for maintaining a low level of the NPM mRNA as well as promoting the commitment of muscle cells to myogenesis. We therefore propose a model whereby the downregulation of the NPM mRNA, mediated by HuR, $\mathrm{KSRP}$ and its associated ribonucleases, is required for proper myogenesis.

\footnotetext{
${ }^{1}$ Department of Biochemistry, Goodman Cancer Center, McGill University, McIntyre Building Room 915B, 3655 Promenade Sir William Osler, Montreal, Quebec, Canada H3G 1Y6. ${ }^{2}$ INSERM, UMR 1037, Centre de Recherche en Cancérologie de Toulouse, 31432 Toulouse, France. ${ }^{3}$ Spanish National Cancer Research Centre (CNIO) Telomeres and Telomerase Group, Molecular Oncology Program, C/ Melchor Fernández Almagro, 3, 28029 Madrid, Spain.

${ }^{4}$ Department of Microbiology, Immunology, and Pathology, Colorado State University, Fort Collins, Colorado 80523-1682, USA. ${ }^{5}$ Department of Neurology and Neurosurgery, McGill University, 3801 University Street, Montreal, Quebec, Canada H3A2B4. 6 National Institute on Aging, National Institutes of Health, Biomedical Research Center, Room 06C226, 251 Bayview Boulevard, Suite 100, Baltimore, Maryland 21224-6825, USA. ${ }^{7}$ Istituto Nazionale Ricerca sul Cancro (IST), Laboratory of Gene Expression Regulation, c/o CBA Building A3, Room 30, Largo R. Benzi, 10, 16132 Genova, Italy. Correspondence and requests for materials should be addressed to I.-E.G. (email: imed.gallouzi@mcgill.ca).
} 
M uscle differentiation, also known as myogenesis, represents a vital process that is activated during embryogenesis and in response to injury to promote the formation of muscle fibres ${ }^{1,2}$. Myogenesis requires the activation of muscle-specific promyogenic factors that are expressed at specific steps of the myogenic process and act in a sequential manner. We and others have demonstrated that the expression of genes encoding some of these promyogenic factors such as MyoD, myogenin and the cyclin-dependent kinase p21 are not only regulated at the transcriptional level but are also modulated posttranscriptionally ${ }^{3-8}$. Indeed, modulating the half-lives of these mRNAs plays an important role in their expression. The RNA-binding protein $\mathrm{HuR}$, via its ability to bind specific AU-rich elements (AREs) in the $3^{\prime}$-untranslated regions ( $3^{\prime}$-UTRs) of these mRNAs, protects them from the AU-rich-mediated decay machinery ${ }^{4,5,7-9}$. This HuR-mediated stabilization represents a key regulatory step that is required for the expression of these promyogenic factors and proper myogenesis.

HuR, a member of the ELAV family of RNA-binding proteins, specifically binds to AREs located in the $3^{\prime}$-UTRs of its target transcripts $^{8-12}$ leading to their stability that in turn enhances the expression of the encoded proteins ${ }^{5,7}$. In addition to mRNA stability, HuR modulates the nucleocytoplasmic movement and the translation of target transcripts ${ }^{13-16}$. Our previous data have indicated that $\mathrm{HuR}$ associates with $M y o D$, myogenin and $p 21$ transcripts only during the fusion step of myoblasts to form myotubes ${ }^{5}$. This finding led to the conclusion that HuR promotes myogenesis by stabilizing these mRNAs specifically at this step. In the same study, however, we showed that depleting HuR from proliferating myoblasts prevented their initial commitment to the differentiation process. These observations indicated that HuR promotes muscle fibre formation by also regulating the expression of target mRNAs during the early steps of myogenesis.

Recently, we discovered that $\mathrm{HuR}$ promotes myogenesis through a novel regulatory mechanism involving its caspasemediated cleavage 4 . As muscle cells are engaged in the myogenic process, a progressive accumulation of $\mathrm{HuR}$ in the cytosol is triggered. In the cytoplasm, HuR is cleaved by caspase 3 at its 226th residue, an Asp (D), generating two cleavage products (HuR-CPs: -CP1, $24 \mathrm{kDa}$ and -CP2, $8 \mathrm{kDa}$ ). These HuR-CPs, generated from $\sim 50 \%$ of cytoplasmic $\mathrm{HuR}$, are required for muscle fibre formation ${ }^{4,8}$. Indeed, while wt-HuR can rescue myogenesis in cells depleted of endogenous HuR, the noncleavable HuRD226A mutant failed to do $\mathrm{so}^{4,8}$. In addition, HuR$\mathrm{CP} 1$, by associating with import factor transportin 2, prevents HuR nuclear import promoting its cytoplasmic accumulation. While these data clearly establish that HuR-CP1 modulates the cellular movement of HuR during myogenesis, the role of HuR-CP2 remains unclear.

HuR is not the only RNA-binding protein involved in the posttranscriptional regulation of promyogenic factors. The KH-type splicing regulatory protein (KSRP) is known to associate, in proliferating myoblasts, with the AREs of the myogenin and $p 21$ mRNAs leading to their rapid decay ${ }^{3}$. By doing so, KSRP participates in ensuring the proliferation of myoblasts and prevents their premature commitment to the myogenic process. KSRP promotes mRNA decay in muscle cells by recruiting ribonucleases such as PARN and members of the exosome complex (for example, EXOSC5) to ARE-containing mRNAs such as myogenin and $p 21$ (refs 3,17 ). It was also suggested that when myoblasts become competent for differentiation, KSRP releases myogenin and $p 21$ mRNAs leading to their stabilization. As a consequence, myoblasts enter myogenesis and fuse to form myotubes $^{3}$. Since at this same step HuR associates with and stabilizes these ARE-bearing mRNAs ${ }^{5,7}$, we concluded that the induction of myogenesis involves both KSRP and HuR that modulate the expression of the same mRNAs in an opposite way but at different myogenic steps.

Surprisingly, however, here we report that in undifferentiated muscle cells, HuR and KSRP do not compete but rather collaborate to downregulate the expression of a common target, the Nucleophosmin (NPM, also known as B23) mRNA. HuR forms a complex with KSRP that is recruited to a U-rich element in the $3^{\prime}$-UTR of NPM mRNA. The HuR/KSRP complex, in collaboration with PARN and the exosome, then destabilizes the NPM mRNA leading to a significant reduction in NPM protein levels. Our data also provide evidence supporting the idea that the $\mathrm{HuR} / \mathrm{KSRP}$-mediated decrease of NPM expression represents one of the main events that helps myoblasts commit to the myogenic process.

\section{Results}

NPM is a HuR-mRNA target in undifferentiated muscle cells. We first identified the mRNAs that depend on HuR for their expression in undifferentiated, $\mathrm{C} 2 \mathrm{C} 12$ cells. Endogenous HuR was depleted (siHuR) or not (siCtr) from these cells and total RNA was then prepared and hybridized to mouse arrays containing 17,000 probe sets of known and unknown expressed sequence tags. Consistent with the fact that HuR acts as a stabilizer for many of its mRNA targets ${ }^{10,12}$, we observed a significant decrease in the steady-state levels of 18 mRNAs in siHuR-treated cells. Surprisingly, however, we found that 12 mRNAs are significantly upregulated in these cells (Supplementary Table 1). While many of these transcripts do not have typical AREs and are not known to modulate myogenesis, these observations suggested that in muscle cells $\mathrm{HuR}$, in addition to stabilizing some of its target mRNAs, could also be involved in the destabilization of other transcripts. Therefore, we investigated further this unexpected possibility and assessed whether this HuR destabilizing activity is part of its promyogenic function. To this end, we chose to study, among these upregulated HuRmRNA targets, the NPM mRNA. NPM was identified as a target of HuR in other cell systems ${ }^{18}$ and its downregulation has been shown to be required for the differentiation of other cell models ${ }^{19,20}$. Hence, moderating NPM expression in muscle cells could be one of the early events through which HuR promotes myogenesis.

First, we validated the results of our microarray data using northern blot analysis. The knockdown of $\mathrm{HuR}$ in $\mathrm{C} 2 \mathrm{C} 12$ cells increased the level of NPM mRNA by twofold while, as previously described $^{5}$, it decreased the level of $M y o D$ mRNA by $>50 \%$ (Fig. 1a). Knocking down HuR in these cells also increased NPM protein levels, while myogenin protein levels were reduced (Fig. 1b). Conversely, overexpressing GFP-HuR protein in C2C12 cells significantly decreased the levels of both NPM mRNA and protein, compared with cells transfected with green fluorescent protein (GFP) only (Supplementary Fig. 1). Together, these observations establish that in undifferentiated muscle cells, HuR prevents the overexpression of NPM mRNA and protein.

HuR-mediated NPM mRNA destabilization promotes myogenesis. If downregulating NPM levels represent an important event during the early steps of myogenesis, decreasing its expression levels in $\mathrm{C} 2 \mathrm{C} 12$ cells should promote their ability to enter this process. To investigate this possibility, we assessed NPM mRNA and protein levels during muscle cell differentiation. We observed that, although, as expected ${ }^{21}$, MyoD mRNA levels increased during this process, the abundance of the NPM mRNA and protein significantly decreased as soon as myogenesis was initiated (Fig. 2a-c). To determine whether decreasing NPM 
a
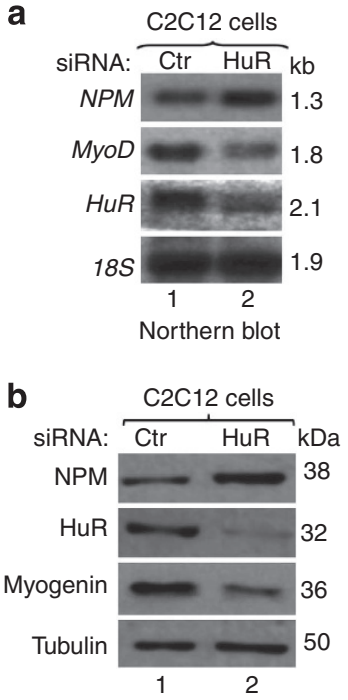

Western blot
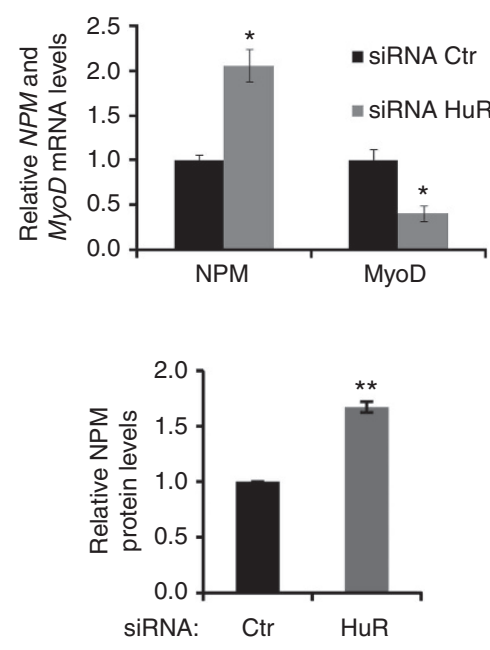

Figure 1 | HuR regulates NPM expression in muscle cells. Exponentially growing $\mathrm{C} 2 \mathrm{C} 12$ myoblasts were transfected with the HuR or control ( $\mathrm{Ctr}$ ) siRNAs. (a) RNA was prepared $48 \mathrm{~h}$ after transfection with $\mathrm{HuR}$ or $\mathrm{Ctr}$ siRNAs. Northern blot was performed using radiolabelled probes against NPM, MyoD, HuR mRNAs and $18 \mathrm{~S}$ (loading control). The band intensities of NPM, MyoD mRNAs and 185 were determined using ImageQuant Software. The NPM and MyoD mRNA levels were normalized on 185 rRNA level. (b) Forty-eight hours after transfection with HuR or Ctr siRNAs, whole-cell extracts were prepared and western blot was performed using antibodies against NPM, HuR, myogenin and $\alpha$-tubulin (loading control). ImageQuant was used to determine the NPM level, normalized on $\alpha$-tubulin level.

In the histograms, the siRNA HuR condition was plotted relative to the siRNA Ctr condition \pm s.e.m. of three independent experiments ${ }^{\star} P<0.01$, ${ }^{\star \star} P<0.001$ ( $t$-test).

expression is required for the initiation of myogenesis, we tested the impact that NPM knockdown (Fig. 2d) could have on the ability of $\mathrm{C} 2 \mathrm{C} 12$ cells, expressing or not $\mathrm{HuR}$, to enter this process. We observed that the depletion of endogenous NPM by small interfering RNA (siRNA) not only triggered the formation of more and larger muscle fibres when compared with siCtr-treated cells, but also re-established myogenesis in HuRknockdown C2C12 cells (Fig. 2d-h and Supplementary Fig. 2). These observations, together with the fact that overexpressing GFP-NPM prevented myogenesis (Supplementary Fig. 3), clearly demonstrate that one way by which HuR promotes the early steps of muscle fibre formation is by downregulating NPM expression.

We have previously shown that HuR is cleaved during muscle cell differentiation into two cleavage products HuR-CP1 $(24 \mathrm{kDa})$ and HuR-CP2 $(8 \mathrm{kDa})$. Our data also indicated that HuR cleavage is a key regulatory event required for proper muscle fibre formation ${ }^{4}$. Since our data (Fig. 2c) have shown that the cleavage of HuR during myogenesis correlates with a decrease in NPM expression, we decided to investigate the effect of HuR-CPs on NPM expression in muscle cells. As expected, we observed that in muscle cells depleted of endogenous HuR, the wild-type (wt$\mathrm{HuR}$ ) but not the non-cleavable isoform of HuR (HuRD226A) was able to re-establish myogenesis as well as the downregulation of the steady-state level of the NPM mRNA. Surprisingly, however, HuR-CP2 but not HuR-CP1 was also able to reestablish, in these cells, the downregulation of NPM mRNA levels and myogenesis similarly to wt-HuR (Fig. 2i-l). While these results suggest a correlation between HuR cleavage and the HuR-

mediated downregulation of the NPM transcript, they clearly indicate that the $\mathrm{C}$ terminus of $\mathrm{HuR}$ could play an important role in this effect.

To identify the mechanisms responsible for the decreased expression of NPM mRNA during myogenesis (Fig. 2a), we first performed a nuclear run-on assay ${ }^{22}$ to assess whether this decrease is due to a change in transcription. Since we did not observe any change in the transcription rate during myogenesis (Supplementary Fig. 4), we concluded that the HuR-mediated downregulation of NPM mRNA expression is likely to occur at the level of mRNA stability. Indeed, a pulse-chase experiment ${ }^{23}$ using the RNA polymerase II inhibitor actinomycin D (ActD) showed that depleting HuR in $\mathrm{C} 2 \mathrm{C} 12$ cells significantly increased, from $\sim 5$ to $>9 \mathrm{~h}$, the half-life of the NPM mRNA (Fig. 3a,b). Of note, treatment of cells with ActD did not affect their viability (Supplementary Fig. 5). These results, together with the fact that HuR does not affect the localization or the translation of the NPM mRNA (Fig. 3c,d), indicate that HuR promotes the formation of muscle fibres by destabilizing the NPM mRNA.

HuR destabilizes NPM mRNA via U-rich elements in the $3^{\prime}$-UTR. $\mathrm{HuR}$ is known to regulate its mRNA targets by interacting with AU-/U-rich elements in their $3^{\prime}$-UTRs ${ }^{9,24}$. Therefore, to define the molecular mechanism by which HuR destabilizes the NPM mRNA, we first assessed whether HuR and NPM mRNA associate in muscle cells. Immunoprecipitation (IP) (Fig. 4a) followed by reverse transcription PCR (RT-PCR; upper panel) or RT-quantitative PCR (RT-qPCR; lower panel) analyses ${ }^{23,25}$ showed that HuR and the NPM mRNA coexist in the same complex in muscle cells. To determine whether this association is direct or indirect, we performed RNA electromobility shift assays (REMSAs) ${ }^{23}$ using recombinant glutathione S-transferase (GST) or GST-HuR proteins and radiolabelled RNA probes corresponding to the entire $5^{\prime}$ - or $3^{\prime}$ UTRs of NPM mRNA (Fig. 4b). We observed that GST-HuR forms a complex only with the $3^{\prime}$ but not the $5^{\prime}$-UTR of NPM mRNA (Fig. 4c). To further define the binding site(s) of HuR, we divided the NPM $3^{\prime}$-UTR into two probes P1 and P2 (Fig. 4b) and observed a strong association of GST-HuR with only the P1 probe (Fig. $4 \mathrm{~d}$ ). Using surface plasmon resonance analysis, we confirmed these associations and showed that GST-HuR is bound to the NPM-3'UTR and P1 probe with higher affinity (dissociation constants $\left(K_{\mathrm{D}}\right)$ of $86 \times 10^{-9}$ and $132 \times 10^{-9} \mathrm{M}$, respectively) than the $\mathrm{P} 2$ probe $\left(K_{\mathrm{D}}=324 \times 10^{-9} \mathrm{M}\right)$ (Fig. $\left.4 \mathrm{e}\right)$. In addition, further subdivision of $\mathrm{P} 1$ and 2 probes into three smaller regions, P1/2-1, -2 and -3 , showed that HuR only binds to the P1-1 region of NPM-3'-UTR (Fig. 4f and Supplementary Fig. 6a,b). Analysis of the secondary structure of the P1-1 region using the mfold prediction software for mRNA folding ${ }^{26}$ indicated the existence of two U-rich elements (E1 and E2) similar to HuR cis-binding motifs previously identified in other mRNA targets ${ }^{11,23,26}$ (Supplementary Fig. 6c). To determine whether E1 and/or E2 elements mediate the binding of HuR to the NPM $3^{\prime}$-UTR, we mutated each one of their uracils (Us; shown as Ts) as well as those of the element separating E1 and E2 to cytosine (C) (Fig. 4g) and performed REMSA experiments as described above. Since mutating each site separately (P1-1-mut1 or -mut2), but not those of the hinge element (P1-1-mut3), equally disrupted the P1-1/HuR complex (Fig. 4h,i), we concluded that the E1 and E2 elements together constitute the minimum U-rich element required for the direct binding of HuR to the NPM-3'-UTR.

Next, we tested whether these U-rich elements are involved in the HuR-mediated destabilization of the NPM mRNA in muscle cells. We generated reporter complementary DNA (cDNA) plasmids expressing the Renilla luciferase (Rluc) in which we inserted, at the $3^{\prime}$ end, either the wild-type NPM $3^{\prime}$-UTR (Rluc-NPM- $3^{\prime}$ ) or the NPM $3^{\prime}$-UTR mutant 1 (Rluc-NPM- $3^{\prime}$ - 

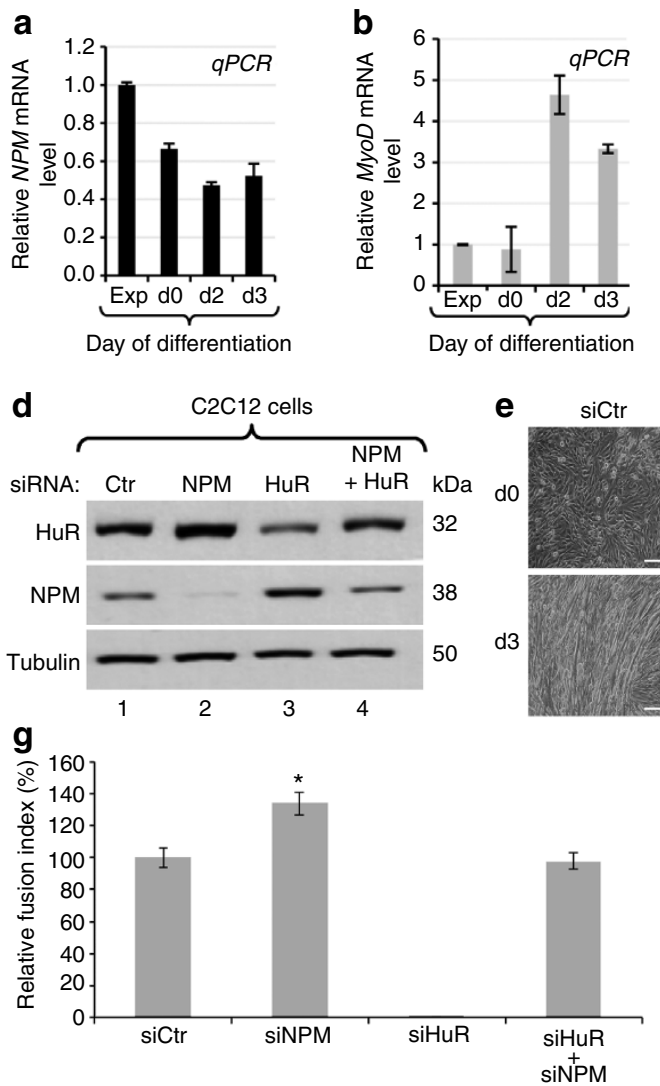

i
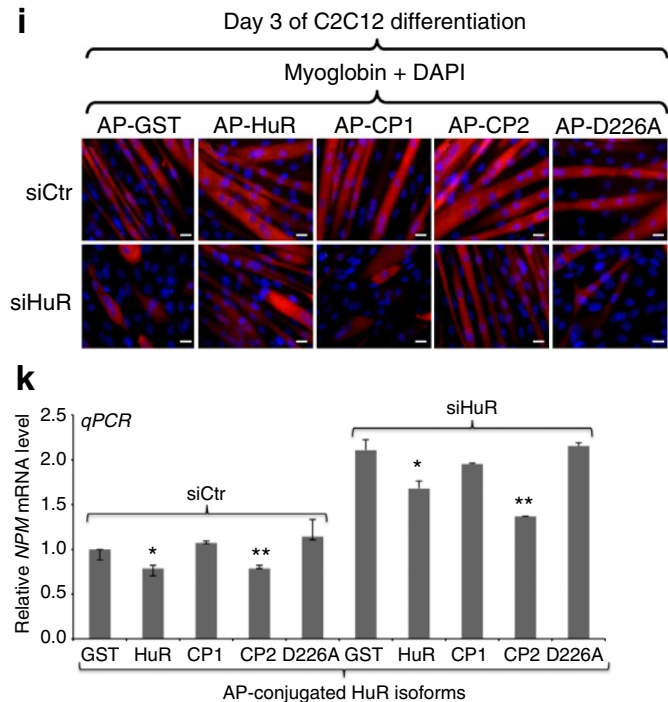

e siCtr
C
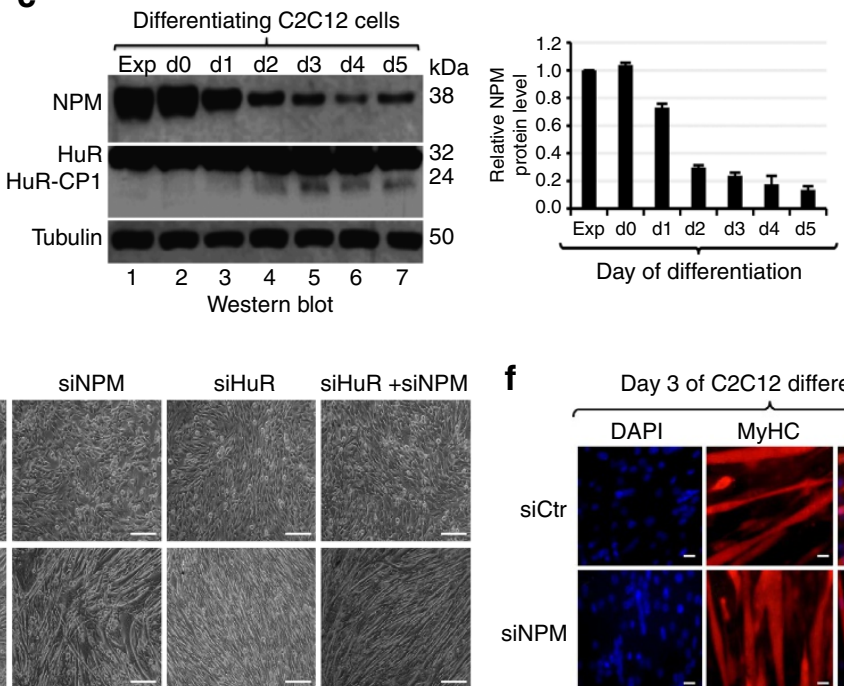

h
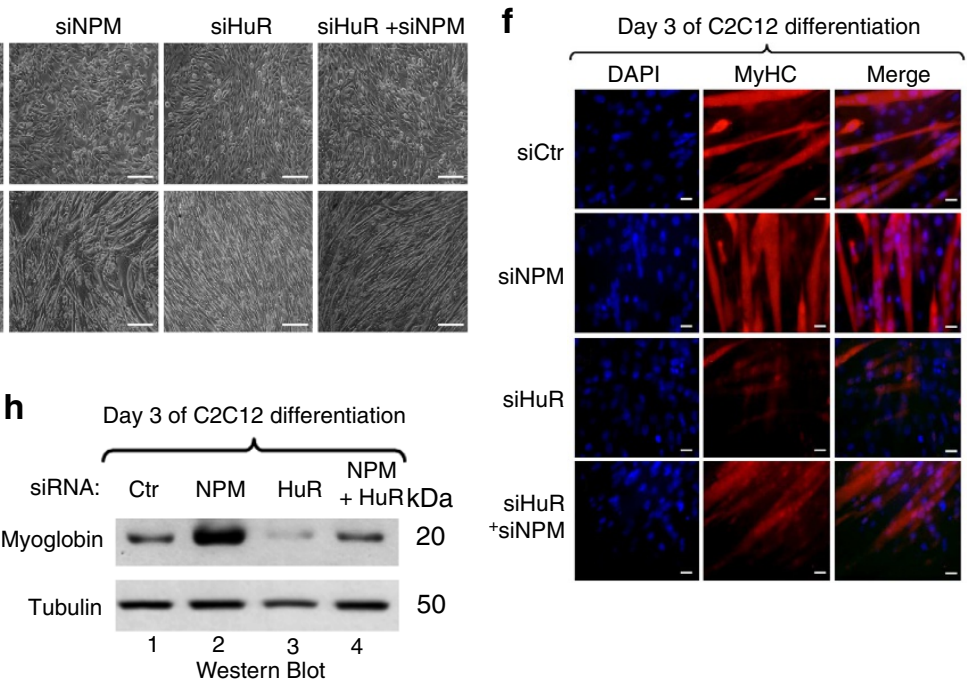
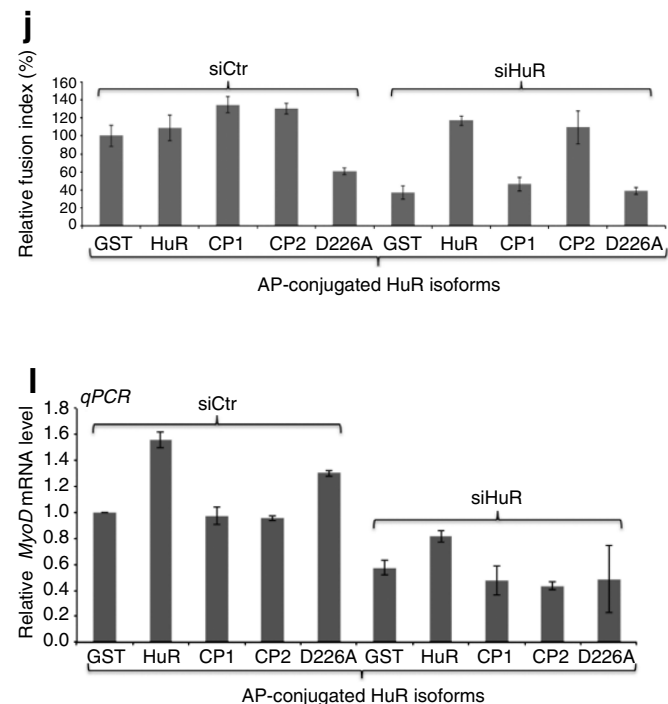

Figure 2 | Reducing NPM level is required for the commitment of muscle cells into the myogenic process. (a,b) Total RNA was isolated from exponentially growing (Exp) or confluent C2C12 myoblasts (day 0) and during the differentiation process (days 2,3). (a) NPM and (b) MyoD mRNA levels, determined by RT-qPCR, were standardized against GAPDH mRNA and expressed relative to the exponential condition. Values were plotted \pm s.e.m. of three independent experiments. (c) Total cell extracts were prepared from growing (Exp) as well as differentiating C2C12 myoblasts (days 1-5) and used for western blot with antibodies against NPM, HuR or $\alpha$-tubulin (loading control). The NPM protein level was determined using the Imagequant software, standardized against tubulin levels and plotted relative to the exponential condition \pm s.e.m. of three independent experiments. (d-h) Knockdown of HuR, NPM and NPM + HuR was performed in C2C12 cells and differentiation was induced $48 \mathrm{~h}$ post treatment with siRNAs. (d) Total cell lysates were prepared $48 \mathrm{~h}$ post transfection and used for western blot with antibodies against NPM, HuR and $\alpha$-tubulin. (e) Phase contrast pictures showing the differentiation status at $\mathrm{dO}$ and d3. Scale bar, $50 \mu \mathrm{m}$. (f) Immunofluorescence experiments were performed using the anti-My-HC antibody and DAPI staining. Images of a single representative field were shown. Scale bar, $10 \mu \mathrm{m}$. (g) The fusion index (calculated from three independent experiments) was determined for muscle fibres shown in $\mathbf{f}$. (h) Total cell extracts were prepared from the cells described above and used for western blot analysis using antibodies against myoglobin and $\alpha$-tubulin. (i-I) C2C12 cells depleted or not of HuR were treated twice (see Methods) with $50 \mathrm{nM}$ AP-GST, -HuR-GST, -CP1-GST, -CP2-GST or HuRD226A-GST and $24 \mathrm{~h}$ after the second treatment with these chimera they were induced for differentiation. (i) Immunofluorescent images and (j) fusion index of fibres fixed on day 3 of differentiation process. Scale bar, $10 \mu \mathrm{m}$. (k,I) NPM (k) and MyoD (I) mRNA levels in confluent myoblasts treated as described above. mRNA levels were plotted relative to the GST- and siCtr-treated conditions \pm s.e.m. of three independent experiments. For histograms in $\mathbf{g}, \mathbf{k},{ }^{\star} P<0.01,{ }^{\star \star} P<0.001$ (t-test). 

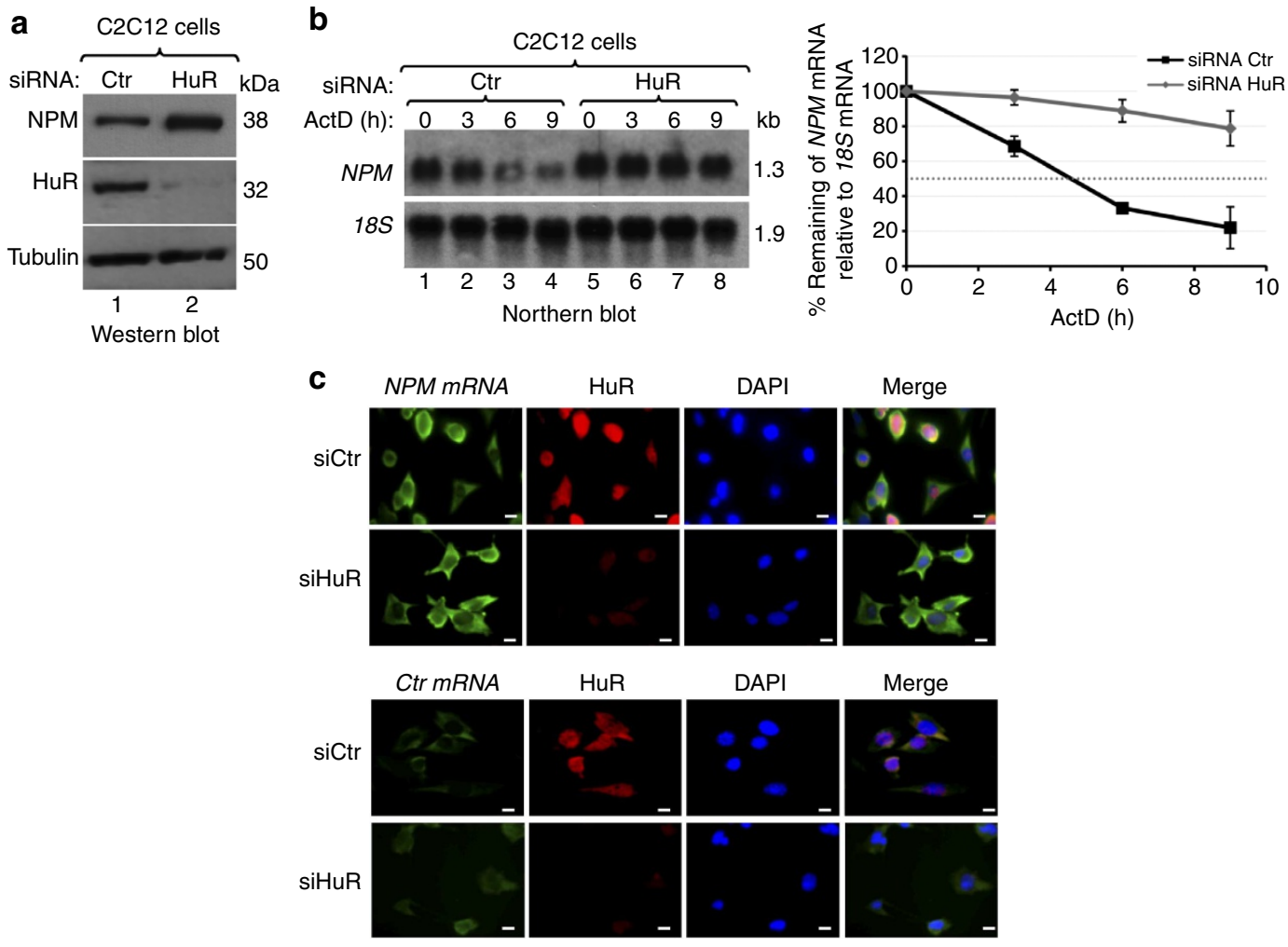

d
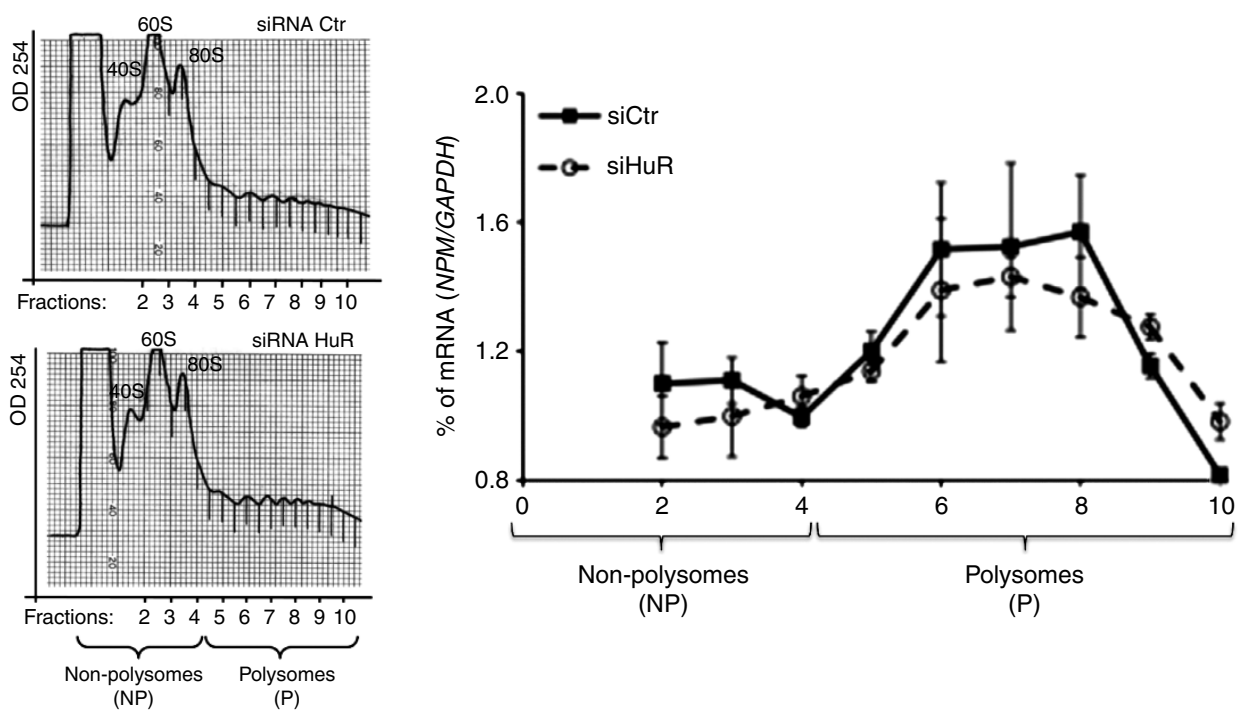

Non-polysomes

(NP)

(P)

Figure 3 | HuR regulates the stability of the NPM mRNA. (a) Total extracts from C2C12 myoblasts treated with siRNA HuR or siRNA Ctr were used for western blot analysis to detect NPM, HuR and $\alpha$-tubulin (loading control). Representative gel of three independent experiments. (b) RNA was prepared from $\mathrm{C} 2 \mathrm{C} 12$ cells transfected with HuR or Ctr siRNAs (for $48 \mathrm{~h}$ ), and then treated with ActD for $0,3,6$ or $9 \mathrm{~h}$. Northern blot analysis was performed using radiolabelled probes against NPM mRNA and 18S rRNA (loading control). NPM and 18S band intensities were measured using ImageQuant and the stability of NPM mRNA was determined relative to the $18 \mathrm{~S}$ for each time point. NPM signal in each one of these time points was compared with NPM level at $\mathrm{O}$ h of ActD treatment, which is considered $100 \%$. These percentages were then plotted \pm s.e.m of three independent experiments. (c) $\mathrm{C} 2 \mathrm{C} 12$ cells transfected with siRNA HuR or siRNA Ctr were fixed, permeabilized and incubated with digoxigenin-labelled in vitro-transcribed antisense probe to detect NPM mRNA ( $\mathrm{a}, \mathrm{e})$ and with sense RNA probe $(\mathrm{i}, \mathrm{m})$ as a control (Ctr probe). Immunofluorescence staining with anti-HuR antibody (b,f,j, $\mathrm{n})$ and with DAPI was performed. A single representative field for each cell treatment is shown. Scale bar, $10 \mu \mathrm{m}$. (d) Forty-eight hours post transfection of exponentially growing $\mathrm{C} 2 \mathrm{C} 12$ cells with siRNA HuR or Ctr, polysomes were fractionated through sucrose gradients (15-50\% sucrose). Absorbance at wavelength $254 \mathrm{~nm}$ was measured to determine the profile of polysome distribution. Ten fractions were collected and divided into two groups: nonpolysome (NP, fractions 1-4) and polysome ( $\mathrm{P}$, fractions 5-10, contain mRNAs engaged in translation) (left panel). RT-qPCR was performed on each fraction using specific primers for NPM and GAPDH mRNAs (right panel). NPM mRNA level was standardized against GAPDH mRNA in each fraction and plotted \pm s.e.m. of three independent experiments. 
mut1, deficient in its ability to bind HuR) (Figs $4 \mathrm{~h}$ and 5a). An IP/ RT-qPCR experiment showed that the association between HuR and the Rluc-NPM-3' mRNA is significantly higher than its association with the control reporters Rluc or Rluc-NPM- $3^{\prime}-$ mut1 (Fig. 5b,c). We next assessed the impact of depleting endogenous
HuR on the expression of the Rluc-NPM-3' mRNA. siCtr- or siHuR-treated $\mathrm{C} 2 \mathrm{C} 12$ cells were transfected with the Rluc, RlucNPM-3' or Rluc-NPM-3'-mut1 plasmids (Supplementary Fig. 7a) and the steady-state levels of these mRNAs were determined by RT-qPCR analysis. While we observed a 3.5-fold increase in the a
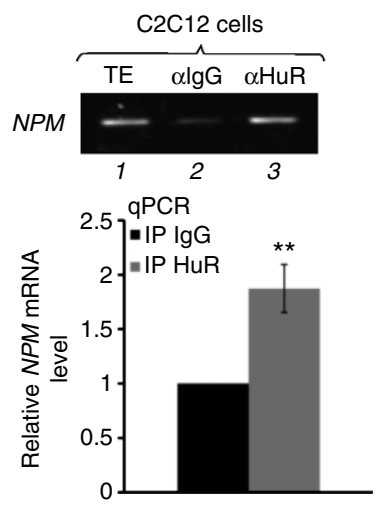

e
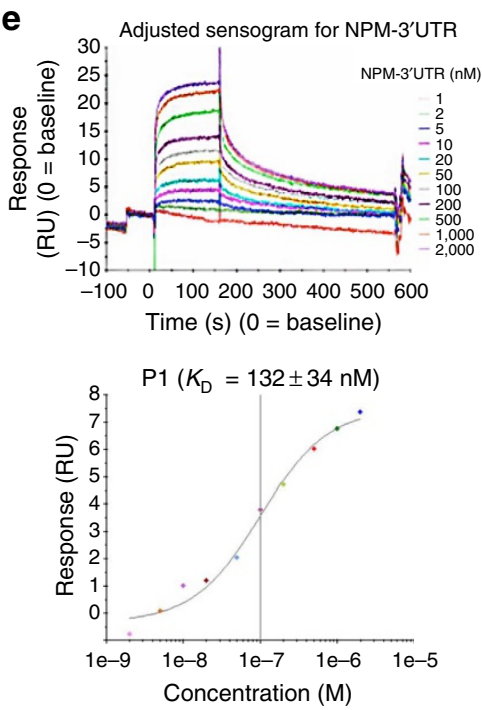

b
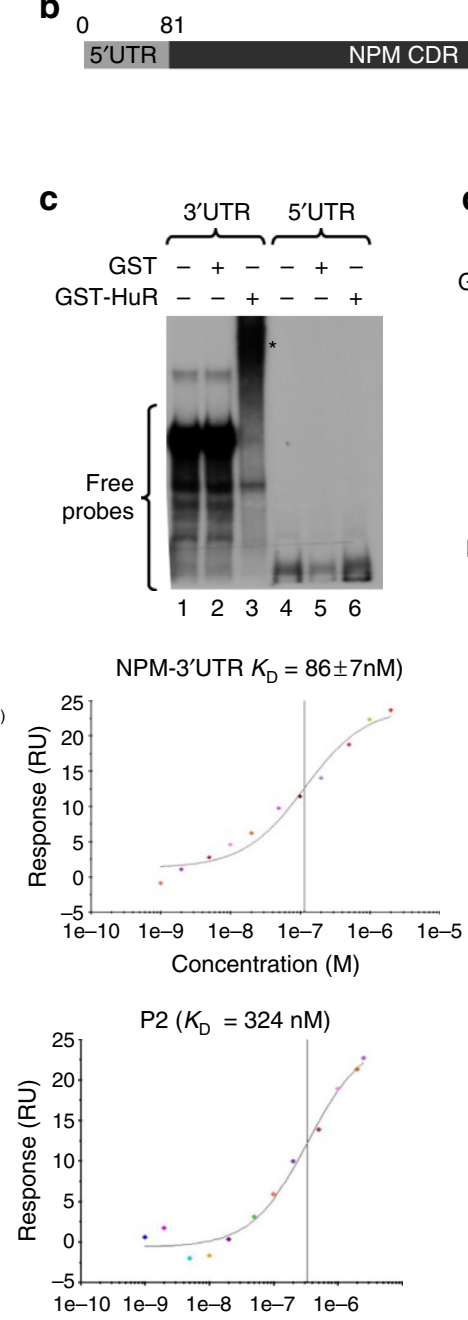

1261

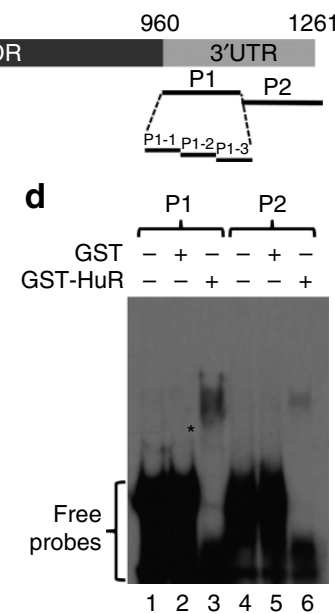

f

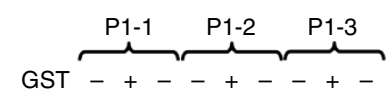

GST-HuR $-\cdots+\cdots+\cdots$

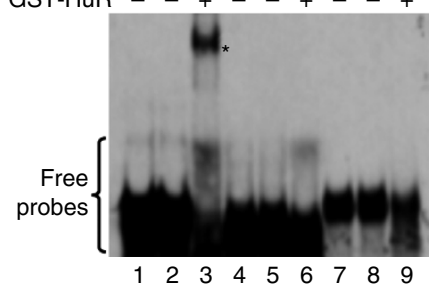

g

E1

E2

P1-1: GAAAAGGGTTTAAACAGTTTGAAATATTC TGTCTTCAT T TCTGTAATAGT TiA

P1-1-mut-1: GAAAAGGGTTTAAACAGC C $\mathcal{*}_{*}$ GAACACCCTGTCT TCATT TCTGTAATAGTTA

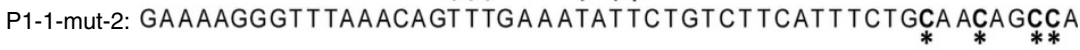

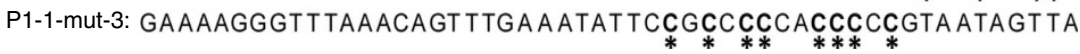
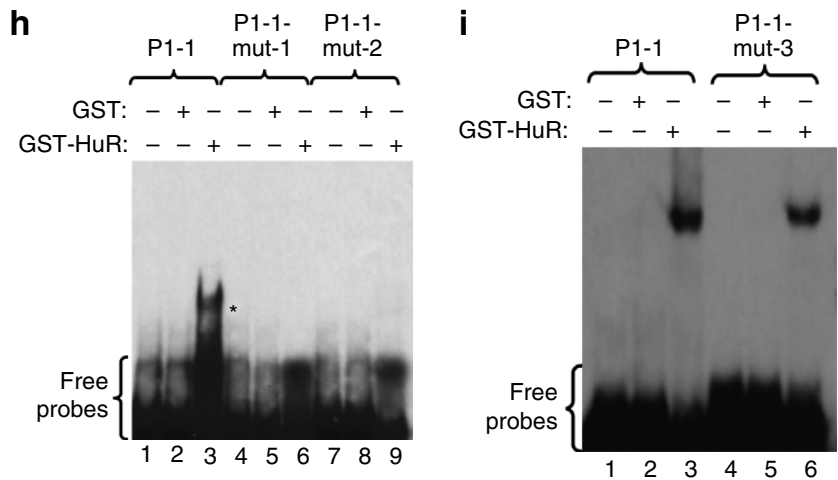
level of the Rluc-NPM-3' mRNA in cells depleted of HuR, but not in those treated with siCtr, the levels of Rluc-NPM-3'-mut1 remained high in both cell types (Fig. 5d). On the other hand, overexpressing GFP-HuR in C2C12 cells decreased the level of the Rluc-NPM-3' mRNA by $>65 \%$ but had no significant effect on the levels of Rluc or Rluc-NPM-3'-mut1 mRNAs (Fig. 5e, Supplementary Fig. 7c). The effect of knocking down or overexpressing HuR on luciferase activity (which is proportional to Rluc protein levels) was similar to those seen for the mRNA levels (Supplementary Fig. 7b,d). Next, we investigated the functional relevance of these HuR-binding sites on NPM mRNA stability. We performed ActD pulse-chase experiments on $\mathrm{C} 2 \mathrm{C} 12$ cells expressing the various Rluc reporters described above and treated or not with $50 \mathrm{nM}$ of recombinant $\mathrm{HuR}$ conjugated to the cell-permeable peptide Antenapedia (AP) ${ }^{14}$ (Fig. 5f). We observed that the half-life of the Rluc-NPM- $3^{\prime}$ mRNA was significantly reduced $(<2 \mathrm{~h})$ only in cells treated with AP-HuRGST but not in those treated with AP-GST ( $>6 \mathrm{~h}$ ). However, the Rluc-NPM-3'-mut1 mRNA remained stable under all treatments (half-life $>6 \mathrm{~h}$ ). While these results clearly demonstrate that an intact HuR-binding site is required for HuR-induced destabilization of the NPM mRNA, it does not provide any indication on their relevance during myogenesis. To address this question, we generated GFP-conjugated full-length wild-type (GFP-NPM-3') or mutated (GFP-NPM-3'-mutl) isoforms of NPM (Fig. 5g). These NPM isoforms were then overexpressed in $\mathrm{C} 2 \mathrm{C} 12$ cells and $48 \mathrm{~h}$ later these cells were induced for differentiation for 3 days. Our experiments showed that overexpressing GFP-NPM-3' reduced the efficiency of muscle fibre formation by $\sim 50 \%$ when compared with GFP alone (Fig. 5h-j). Interestingly, the overexpression of the GFP-NPM-3'mutl isoform, which does not bind HuR, provided a much higher inhibition of myogenesis efficiency than GFP-NPM-3' (>70\%). Together, these results show that the recruitment of HuR to the E1 and E2 U-rich elements of NPM 3'-UTR is required for NPM downregulation and the promotion of myogenesis.

HuR destabilizes the NPM mRNA in a KSRP-dependent manner. It has been shown that during myogenesis, transcripts such as myogenin and $p 21$ but not MyoD are destabilized by KSRP in proliferating myoblasts ${ }^{3}$. However, at later stages (fusion step), these mRNAs are stabilized by HuR, leading to an increase in their expression levels and to the promotion of myotube formation ${ }^{5,7}$. Therefore, since KSRP and HuR target common transcripts in muscle cells ${ }^{3,5,7}$ and our observation showed that depleting the expression of either of these two proteins in C2C12 cells equally prevented myogenesis (Supplementary Fig. 8), we tested whether KSRP could be implicated in the HuR-mediated destabilization of the NPM mRNA in proliferating C2C12 cells.
We first determined the effect KSRP depletion could have on the expression level of the NPM mRNA and protein. We showed that, similarly to HuR depletion, the knockdown of KSRP significantly increased the expression levels of NPM mRNA ( $>2$-fold) and protein ( $>1.5$-fold) (Fig. 6a,b). In addition, ActD pulse-chase experiments indicated that the knocking down KSRP increased the half-life of NPM mRNA to a similar extent than HuR (compare Fig. 3b with Fig. 6c). However, as expected ${ }^{3}$, KSRP knockdown had no effect on the stability of the $M y o D$ mRNA, while it increased the half-life of $p 21$ mRNA from $\sim 3.6$ to $>6 \mathrm{~h}$ (Fig. 6c). Altogether, these data show that both HuR and KSRP decrease the expression level of the NPM protein by shortening the half-life of the NPM mRNA. Surprisingly, we additionally observed that overexpressing KSRP alone in $\mathrm{C} 2 \mathrm{C} 12$ cells, unlike HuR, does not affect NPM mRNA or protein levels (Fig. 6d,e) despite promoting the decreased expression of p21. Since knocking down KSRP by $>90 \%$ prevented the HuR-mediated decrease of the NPM mRNA and protein (Fig. 6f-h), we concluded that, although KSRP alone is not able to destabilize NPM mRNA, KSRP is required for the HuR-mediated downregulation of NPM level in muscle cells.

To gain insight into the molecular mechanisms by which $\mathrm{HuR}$ and KSRP affect the fate of NPM mRNA in muscle cells, we first determined whether KSRP also associates with this transcript. IP experiments using the anti-KSRP antibody ${ }^{17}$ followed by RT-PCR and -qPCR analyses showed that KSRP binds to the NPM mRNA similarly to HuR (Figs 4a and 7a). REMSA using total extracts prepared from $\mathrm{C} 2 \mathrm{C} 12$ cells that were incubated with radiolabelled $N P M-3^{\prime}-\mathrm{UTR}$ or the P1 or P2 probes (Fig. $4 \mathrm{~b}$ ) and with the anti-KSRP antibody showed that, similarly to $\mathrm{HuR}$ (Fig. 4), KSRP associates with the $3^{\prime}$-UTR of NPM through the P1 but not the P2 region (Fig. 7b). To determine the KSRP-binding site(s) in the NPM 3'-UTR, we incubated the recombinant KSRP with intact or mutated radiolabelled P1 probes and performed the same REMSA experiments described in Fig. 4. KSRP formed complexes with P1-1 and P1-1-mut2 but not with P1-1-mut1 (mut-E1) probes (Fig. 7c). In addition, IP experiments with antiKSRP antibody were performed on cells expressing the Rluc reporters described in Fig. 5a. We observed that, while KSRP associated with the Rluc-NPM- $3^{\prime}$ mRNA and Rluc-NPM-3'-mut2, it failed to interact with the Rluc-NPM-3'-mut1 message (Fig. $7 \mathrm{~d}$,e and Supplementary Fig. 9). Therefore, together these results show that KSRP directly associates with the NPM mRNA through the E1 element that is also one of the sites that mediates HuR binding.

Next, we investigated whether HuR and KSRP can form a complex. IP experiments on cell extracts treated or not with $100 \mu \mathrm{g} \mathrm{ml}^{-1}$ RNAse A indicated that HuR and KSRP associate in $\mathrm{C} 2 \mathrm{C} 12$ cells in an RNA-independent manner (Fig. 7f,g).

Figure 4 | HuR binds to the NPM mRNA via two U-rich sequences within the $\mathbf{3}^{\prime}$-UTR. (a) IP experiments were performed using the monoclonal HuR antibody (3A2), or IgG as a control, on total cell lysates from C2C12 cells. RNA was isolated from the immunoprecipitate, and RT-PCR or RT-qPCR was performed using primers specific for NPM and RPL32 mRNAs. The agarose gel (upper panel) shown is representative of three independent experiments. For RT-qPCR (lower panel), NPM mRNA levels were standardized against RPL32 mRNA levels. The normalized NPM mRNA levels were plotted relatively to the IgG IP condition \pm s.e.m. of three independent experiments. ${ }^{\star \star} P<0.001$ ( $t$-test). (b) Schematic representation of the NPM mRNA sequence. The probes covering the NPM 3'-UTR (P1, P2, P1-1 to P1-3) used to generate radiolabelled RNA probes for REMSAs are indicated (black lanes). (c,d,f,h,i) Gel-shift binding assays were performed by incubating $500 \mathrm{ng}$ of purified GST or GST-HuR protein with the radiolabelled cRNA (c) $3^{\prime}-$ UTR and 5'-UTR, (d) P1 and P2, (f) P1-1 to P1-3, (h) P1-1, P1-1-mut1 and P1-1-mut2 and (i) P1-1 and P1-1-mut3 probes. These gels are representative of three independent experiments. *shown in panels $4 \mathrm{c}, \mathrm{f}$ and $\mathrm{h}$ indicate the location of shifted complex. (e) BIACORE, a surface plasmon resonance-based biosensor technology, was used for a kinetic binding study between GST-HuR and NPM 3'-UTR, P1 or P2 probes. GST-HuR was captured on a Series S CM5 chip and increased concentrations of NPM cRNA probes, as indicated, were injected over the surface. Injections were performed for $150 \mathrm{~s}$ to measure association, followed by a $400 \mathrm{~s}$ flow of running buffer to assess dissociation. The association/dissociation ratio of GST-HuR to NPM $3^{\prime}$-UTR is shown in the sensorgram (top left panel). The binding affinity $\left(K_{D}\right)$ of GST-HuR to NPM 3'-UTR (top right panel), P1 or P2 (bottom panels) cRNA probes is also shown. (g) Nucleotide sequence of probe P1-1 showing the thymidine $(T)$ residues that were mutated to cytidine $(C)$ residues (identified by asterisks) to generate the P1-1-mut1, P1-1-mut2 and P1-1-mut3. The sequences highlighted by the dashed boxes as E1 and E2 are the single-strand AU-rich sequences identified using mfold software. 
a

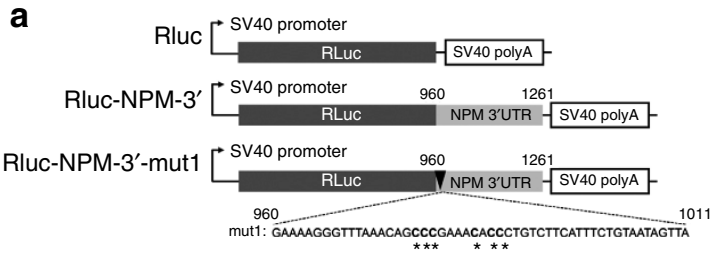

C

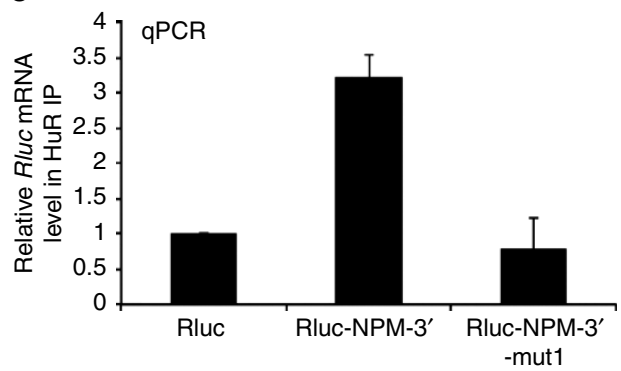

b
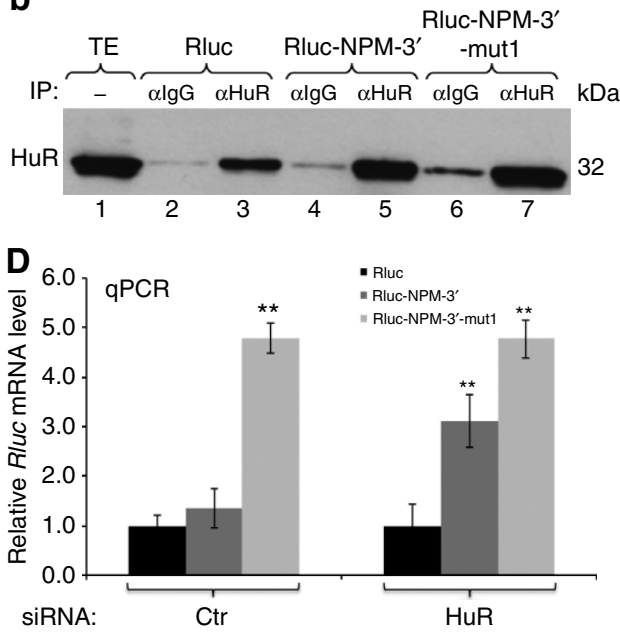

f

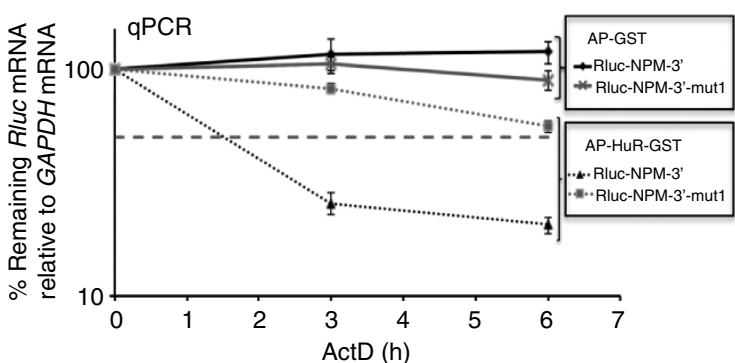

h

Day 3 of $\mathrm{C} 2 \mathrm{C} 12$ differentiation

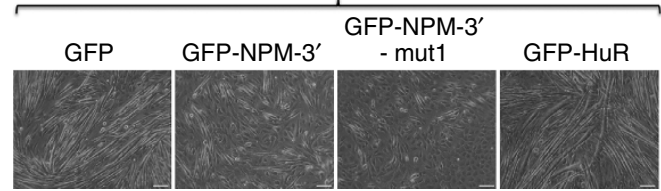

j

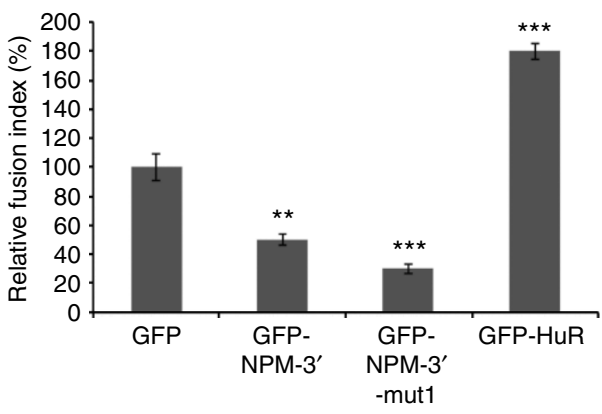

Figure 5 | An intact P1-1 element is required for HuR-mediated regulation of NPM expression. (a) Schematic diagram of the Renilla luciferase reporters used in the experiments described in a-f. (b,c) Exponentially growing C2C12 cells were transfected with plasmids expressing Rluc, Rluc-NPM-3' or Rluc-NPM- $3^{\prime}-$ mut1. Total extracts from these cells were prepared $24 \mathrm{~h}$ after transfection and used for IP using the anti-HuR antibody (3A2) or lgG as a control. (b) Western blot analysis with the anti-HuR antibody was performed using these IP samples. (c) Rluc mRNA associated with the immunoprecipiated HuR was determined by RTqPCR. The relative Rluc RNA levels from Rluc-NPM-3' and Rluc-NPM-3'-mut1 were plotted relatively to the Rluc RNA reporter \pm s.e.m. of three independent experiments. (d,e) The expression levels of these Rluc RNA reporters were also determined by RT-qPCR in C2C12 cells depleted or not of HuR (d) or overexpressing GFP or GFP-HuR (e). The expression levels of the Rluc mRNAs were normalized over total Rluc DNA transfected as described ${ }^{59}$ and then standardized against GAPDH mRNA level \pm s.e.m. of three independent experiments ${ }^{\star \star} P<0.001$; NS, non significant $(t$-test). (f) $C 2 C 12$ cells were transfected with the Rluc reporter RNA described above in the presence of AP-GST or AP-HuR-GST proteins. The stability of these reporter Rluc mRNAs was determined by AcD pulse-chase experiments. The percentages shown were plotted \pm s.e.m of three independent experiments. (g) Schematic diagram of GFP or the GFP-conjugated full-length NPM isoforms: WT NPM (GFP-NPM-3') and the NPM $3^{\prime}$-UTR with the first AU-rich sequence mutated (GFP-NPM-3'-mut1). The coding region of NPM is shown as NPM CDR. (h-j) C2C12 cells expressing these isoforms or the GFP-HuR protein were fixed on day 3 of muscle cell differentiation. (h) Phase contrast images of the myotubes described above. (i) Immunofluorescence (using the anti-My-HC antibody) images of these myotubes. (j) Fusion index \pm s.e.m of three independent experiments, ${ }^{\star \star} P<0.001,{ }^{\star \star \star} P<0.0001$ (t-test). Images of a single representative field are shown. Scale bar, $50 \mu \mathrm{m}(\mathbf{h})$ and $10 \mu \mathrm{m}(\mathbf{i})$. 
a

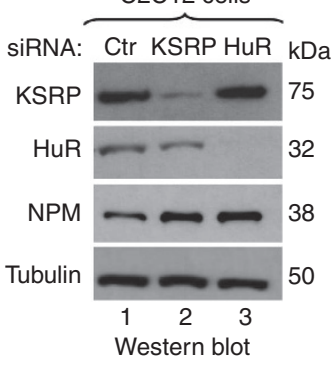

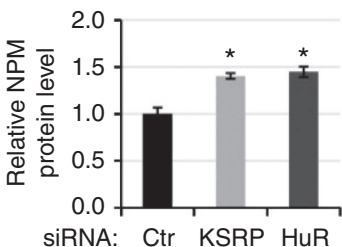

SIRNA: Ctr KSRP HuR b

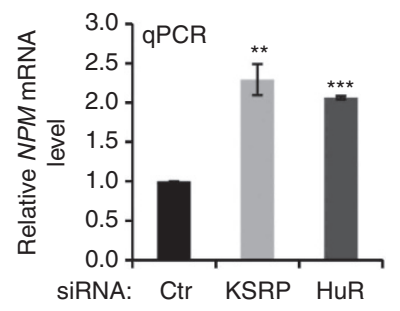

C

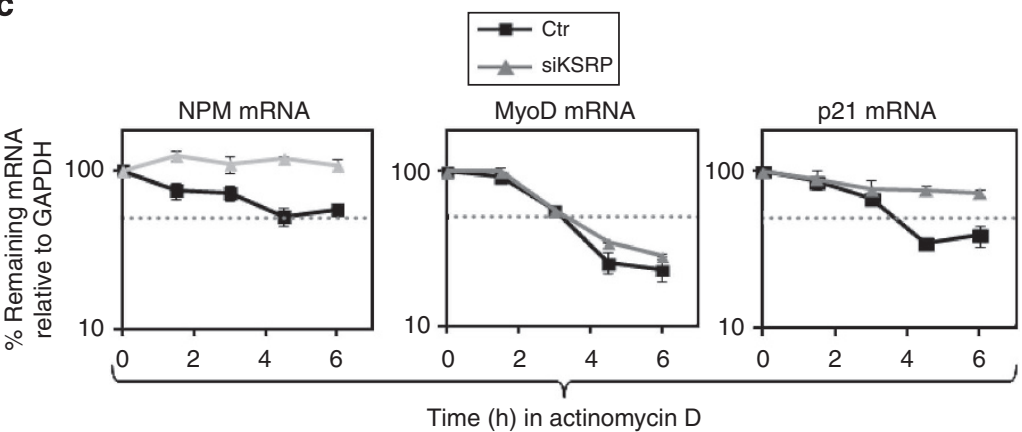

d
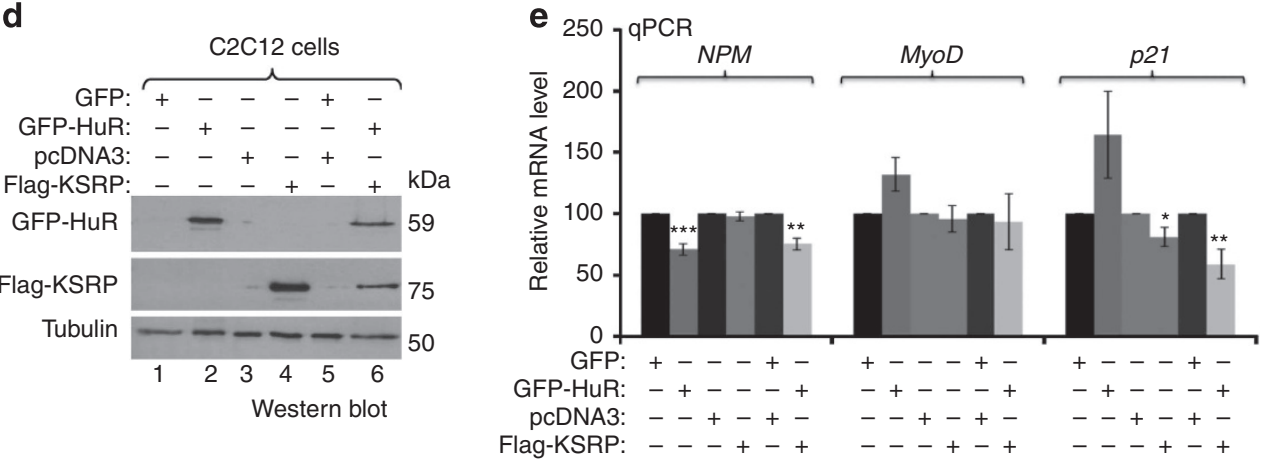

f

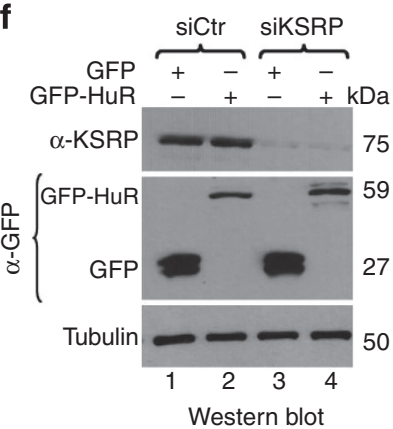

g

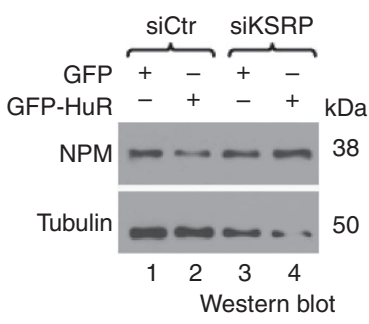

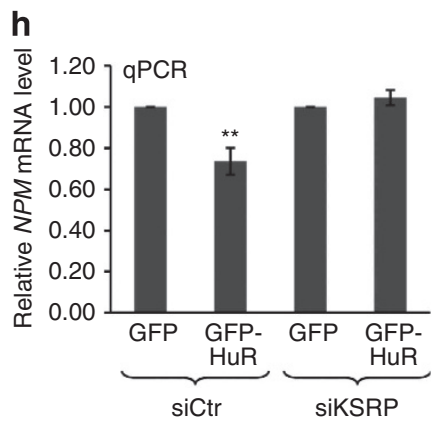

Figure 6 | KSRP is required for the HuR-mediated destabilization of NPM mRNA. (a,b) Exponentially growing C2C12 cells were treated with siRNA Ctr or siRNA against HuR or KSRP. Total cell (a) or RNA (b) extracts from these cells were prepared $48 \mathrm{~h}$ after transfection. (a) Western blot analysis was performed using antibodies to detect KSRP, NPM, HuR and $\alpha$-tubulin (loading control). (b) RT-qPCR analysis was performed to assess NPM mRNA levels that were standardized against GAPDH mRNA. The levels of NPM protein (a) or mRNA (b) in cells depleted of HuR or KSRP were plotted relative to the siRNA Ctr condition \pm s.e.m. of three independent experiments. (c) The stability of the NPM, MyoD and p21 mRNA in C2C12 cells depleted or not of KSRP was determined by AcD pulse-chase experiments. RT-qPCR analysis was performed using specific primers for these three mRNAs. mRNA levels were then standardized against GAPDH mRNA and plotted \pm s.e.m of three independent experiments. (d,e) Exponentially growing $\mathrm{C} 2 \mathrm{C} 12$ cells were transfected with the pcDNA3, pcDNA-Flag-KSRP, GFP and GFP-HuR. (d) Total cell extracts or (e) RNA extracts from these cells were prepared $24 \mathrm{~h}$ post transfection. (d) Western blot was performed using antibodies against Flag, HuR or $\alpha$-tubulin (loading control). (e) RT-qPCR analysis was performed as described above in $\mathbf{c}$ and mRNA levels were plotted relative to their levels in control cells \pm s.e.m. of three independent experiments. (f-h) GFP and GFP-HuR plasmids were transfected in C2C12 cells depleted or not of KSRP. Twenty-four hours later total protein extracts and RNA were prepared. $(\mathbf{f}, \mathbf{g})$ Western blot analysis was performed with antibodies against (f) KSRP, GFP and (g) NPM. $\alpha$-tubulin levels were assessed as a loading control in both $\mathbf{f}$ and $\mathbf{g}$. (h) RT-qPCR analysis was performed as described above. NPM mRNA levels were standardized against GAPDH mRNA level. In each condition, the level of NPM mRNA in siKSRP-treated cells was plotted relative to its levels in siRNA Ctr-treated and GFP-transfected cells \pm s.e.m. of three independent experiments. In the histograms presented in $\mathbf{a}, \mathbf{b}, \mathbf{e}, \mathbf{h},{ }^{\star} P<0.01,{ }^{\star \star} P<0.001,{ }^{\star \star \star} P<0.0001(t$-test). 
a
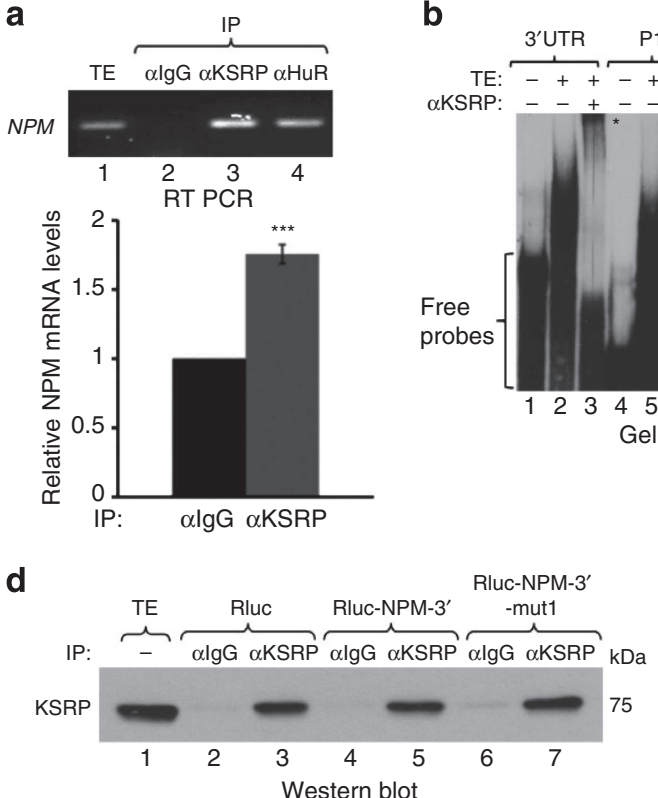

b

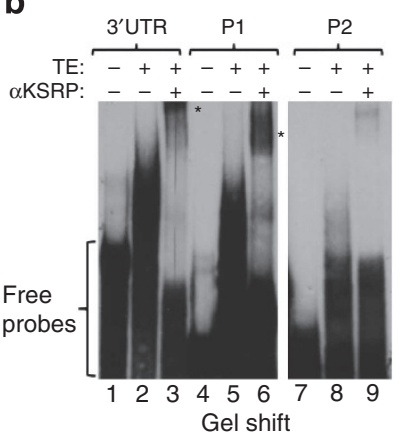

C

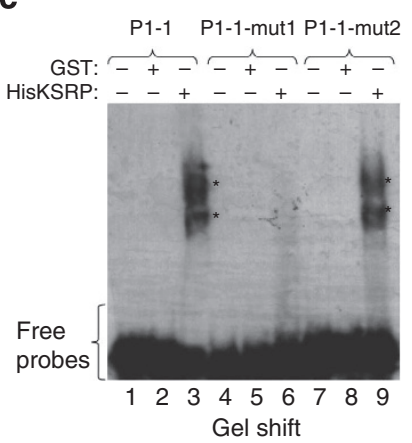

e

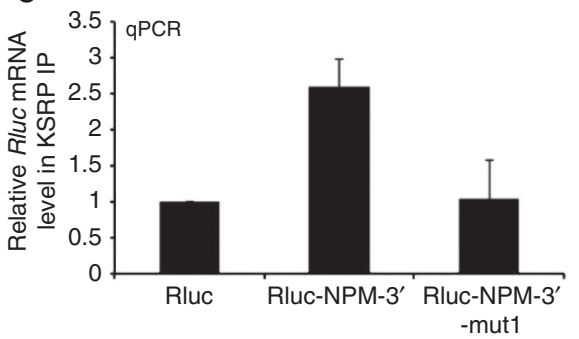

f

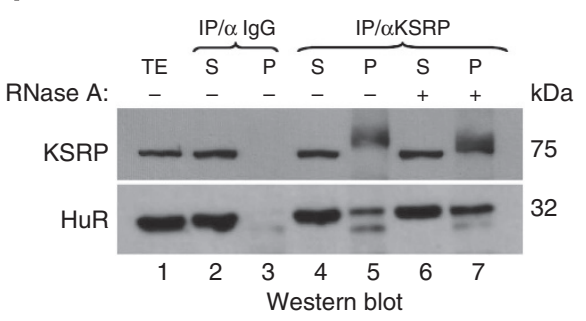

g

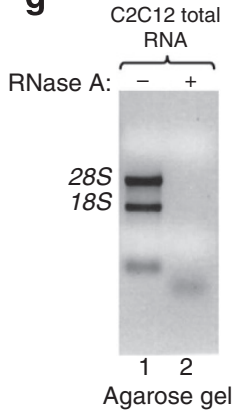

h $\quad$ GST

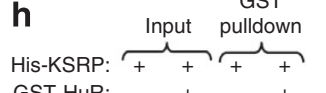

GST-HuR: - + - +

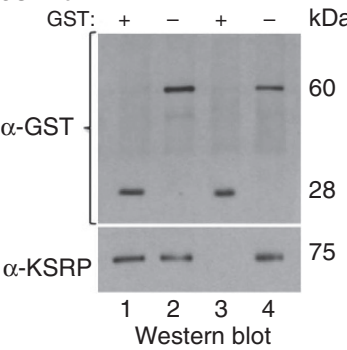

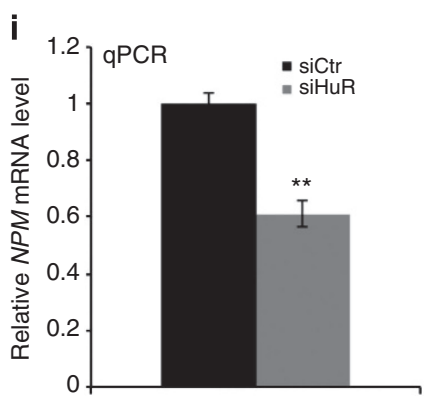

IP KSRP

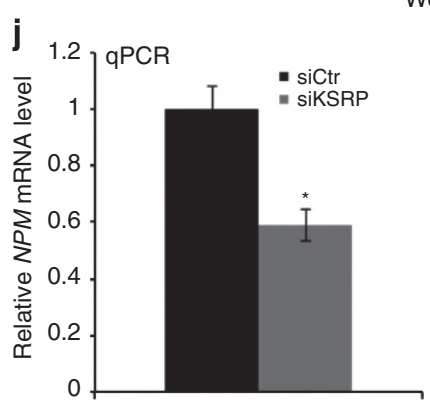

IP HuR

Figure 7 | HuR and KSRP form a complex and bind to the same element in the NPM $\mathbf{3}^{\prime}$-UTR. (a) IP coupled to RT-PCR (upper panel) or RT-qPCR (lower panel) experiments was performed to determine the association of KSRP and HuR with the NPM mRNA in C2C12 cells. For RT-qPCR, NPM mRNA levels are shown \pm s.e.m. of three independent experiments, ${ }^{\star \star \star} P<0.0001$ ( $t$-test). (b) Supershift binding assay was performed to demonstrate that the radiolabelled NPM $3^{\prime}$-UTR as well as the P1 cRNA probe, unlike the P2 probe, can associate to a complex containing KSRP (indicated by an asterisk (*)). (c) Gel-shift binding assay was performed with $500 \mathrm{ng}$ of purified GST or His-KSRP proteins and the indicated radiolabelled cRNA probes. *shown on gel indicates the location of shifted complexes. (d,e) IP experiments were performed on C2C12 cells expressing Rluc, Rluc-NPM-3' and Rluc-NPM-3'-mut1 using the KSRP or IgG antibody. IP of KSRP (d) and association with reporter RNAs (e) were determined as described in Fig. 5b,c. (f) C2C12 extracts treated or not with RNase A were used for IP experiments with the anti-KSRP or -lgG antibodies. The binding of HuR to KSRP was then assessed by western blot. For unknown reasons, we observed a shift in the molecular weight of KSRP that we believe could be due to the IP of a post-translationally modified KSRP isoform. (g) Agarose gel demonstrating effectiveness of RNAse treatment. (h) In vitro GST pull-down assay demonstrating that HuR directly interacts with KSRP. Input lanes account for $10 \%$ of the reaction performed in the assay. (i,j) IP coupled to RT-qPCR experiments was performed using anti-KSRP (i) or HuR (3A2) (j) antibodies on total extract from C2C12 cells treated with siHuR (i) or siKSRP (j). NPM mRNA levels in the immunoprecipitates were standardized (as described in the Methods) and normalized to the corresponding IgG and input sample. The NPM mRNA levels in siHuR or siKSRP conditions were plotted relative to siCtr conditions \pm s.e.m. of three independent experiments, ${ }^{\star} P<0.01$, ${ }^{\star \star} P<0.001$ ( $t$-test). All gels/blots shown in the figure are representative of three independent experiments (except for the gel shown in Fig. $7 \mathrm{~b}$, which is representative of two). 

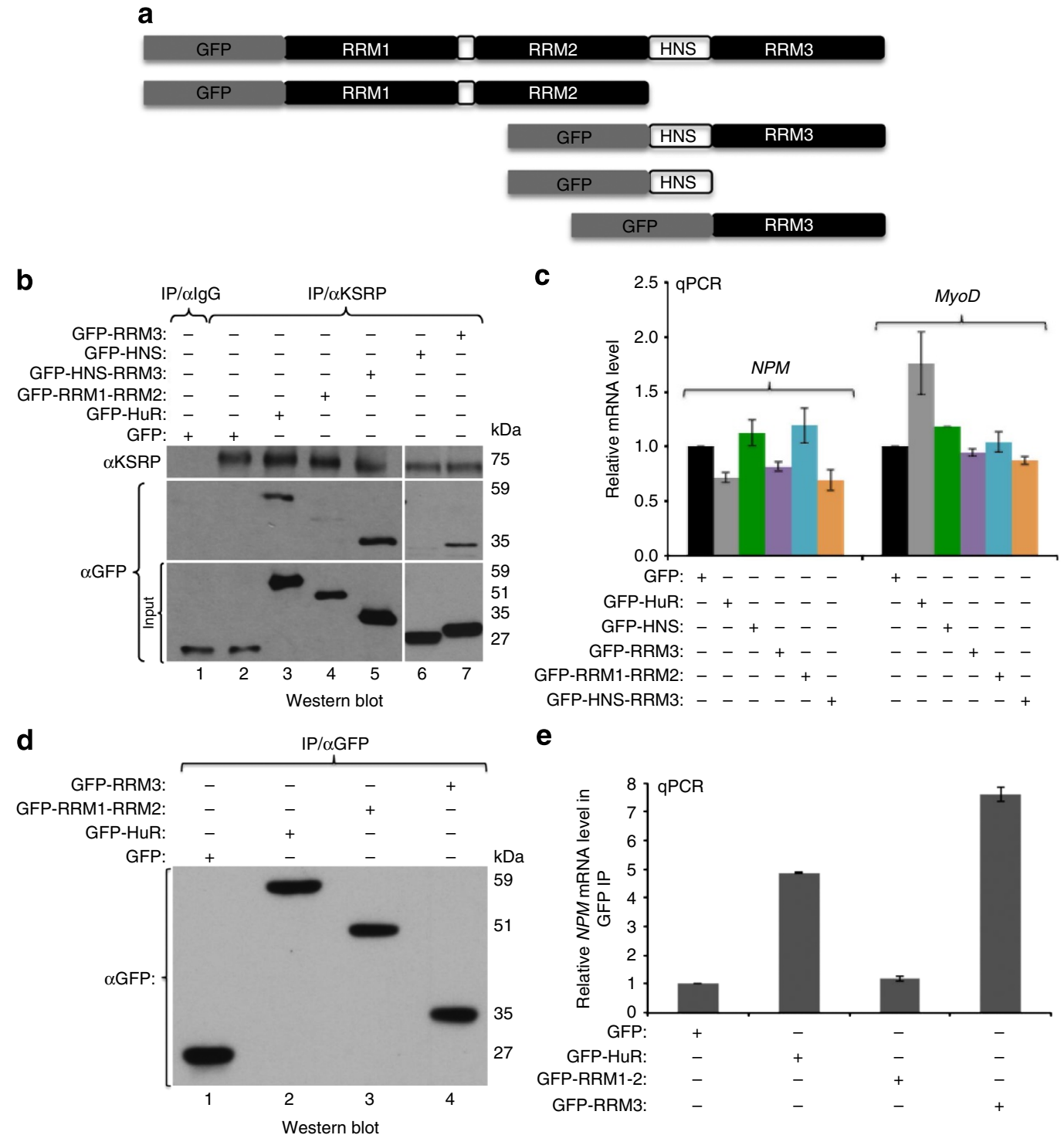

e

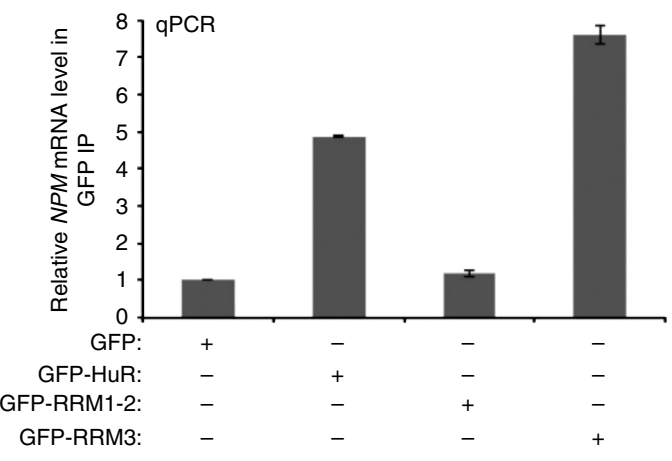

Figure 8 | The HuR-RRM3 motif is required for the formation of KSRP/HuR complex. (a) Schematic diagram depicting the primary structure of HuR protein shows the three RNA-binding domains (RRM1-3) and the HNS. Also shown are the different HuR isoforms conjugated to the GFP tag. (b-e) Exponentially growing C2C12 muscle cells were transfected with GFP, GFP-HuR, GFP-RRM1-RRM2, GFP-HNS-RRM3, GFP-HNS or GFP-RRM3 plasmids. Total cell extracts $(\mathbf{b}, \mathbf{d})$ or total RNA $(\mathbf{c}, \mathbf{e})$ were prepared from these cells $24 \mathrm{~h}$ post transfection. (b) IP experiments on these cell extracts were performed using the KSRP antibody or IgG as a control. The input (10\% of the total extract) and the immunoprecipitate were analysed by western blot using antibodies against GFP. (c) RT-qPCR analysis of total RNA prepared from these cells was performed using specific primers for NPM, MyoD and GAPDH mRNAs. NPM and MyoD mRNA levels were standardized against GAPDH mRNA and plotted relative to the GFP control condition \pm s.e.m. of three independent experiments. (d) IP experiments were performed using the GFP antibody on extracts from the C2C12 cells expressing GFP, GFP-HuR, GFP-RRM1-RRM2 or GFP-RRM3 and analysed by western blot using the GFP antibody. (e) RNA was isolated from the IP as described in d and RT-qPCR was performed using primers specific for NPM and RPL32 mRNAs. NPM mRNA levels were standardized against RPL32 mRNA levels. For each IP sample, NPM mRNA levels were normalized as described in Fig. 5 and plotted relative to the GFP IP \pm s.e.m. of three independent experiments.

However, this RNA-independent interaction between HuR and KSRP was not seen using a lower dose of RNAse A $\left(12.5 \mu \mathrm{g} \mathrm{ml}^{-1}\right)$. Moreover, GST pull-down experiments, using recombinant His-KSRP and GST-HuR, further confirmed this direct interaction (Fig. 7h). We then assessed whether the association between the NPM mRNA and HuR or KSRP requires an intact HuR/KSRP complex. IP/RT-qPCR experiments showed that depleting $\mathrm{HuR}$ from $\mathrm{C} 2 \mathrm{C} 12$ cells significantly decreased ( $>40 \%$ ) the amount of NPM mRNA that binds to KSRP (Fig. 7i). Similarly, although knocking down KSRP in C2C12 cells did not affect the binding of $\mathrm{HuR}$ to the myogenin and p21 mRNAs (Supplementary Fig. 10), it decreased its association with the
NPM mRNA (Fig. 7j). Taken together, these data suggest that in muscle cells the HuR/KSRP complex assembles in an RNAindependent manner and is recruited to the P1-1 element, thus promoting the rapid destabilization of the NPM mRNA.

To gain insight into the mechanism by which HuR executes these functions, we first determined the HuR domain responsible for this activity. We generated plasmids expressing GFP-tagged wild-type HuR or various deletion mutants of HuR (Fig. 8a). Each one of these isoforms was then expressed in $\mathrm{C} 2 \mathrm{C} 12$ cells that were subsequently used for IP experiments with the anti-KSRP antibody. Western blot analysis with the anti-GFP antibody has shown that, similarly to wt-HuR, polypeptides harbouring the 
RRM3 motif (GFP-HNS-RRM3 and GFP-RRM3), but not those harbouring RRM1-2 or HuR nucleocytoplasmic shuttling (HNS) alone, were able to associate with KSRP (Fig. 8b) and promote a significant decrease in NPM mRNA level (Fig. 8c). In addition, an IP/RT-qPCR experiment on cells expressing GFP-HuR, -RRM1-2 or -RRM3 indicated that, while RRM1-2 did not show a strong association with the NPM mRNA, RRM3 associated more with NPM message than wt-HuR (Fig. 8d,e). Since, during myogenesis, cytoplasmic HuR undergoes caspase-mediated cleavage generating HuR-CP1 (24 kDa, RRM1-RRM2- $\left.\Delta \mathrm{HNS}_{1}\right)$ and HuR-CP2 $\left(8 \mathrm{kDa}, \Delta \mathrm{HNS}_{2}-\mathrm{RRM} 3\right)^{4}$ (Fig. $2 \mathrm{C}$ and Supplementary Fig. 11a), we next verified the effect of these CPs on NPM expression. Our a

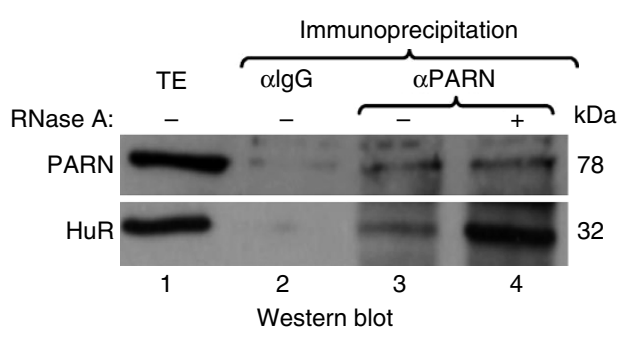

c

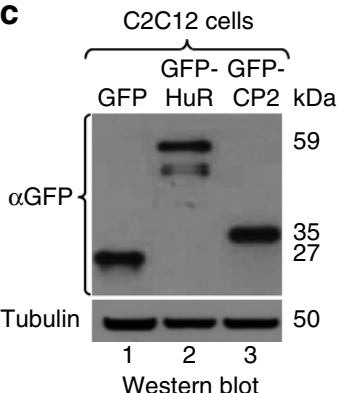

\section{b}
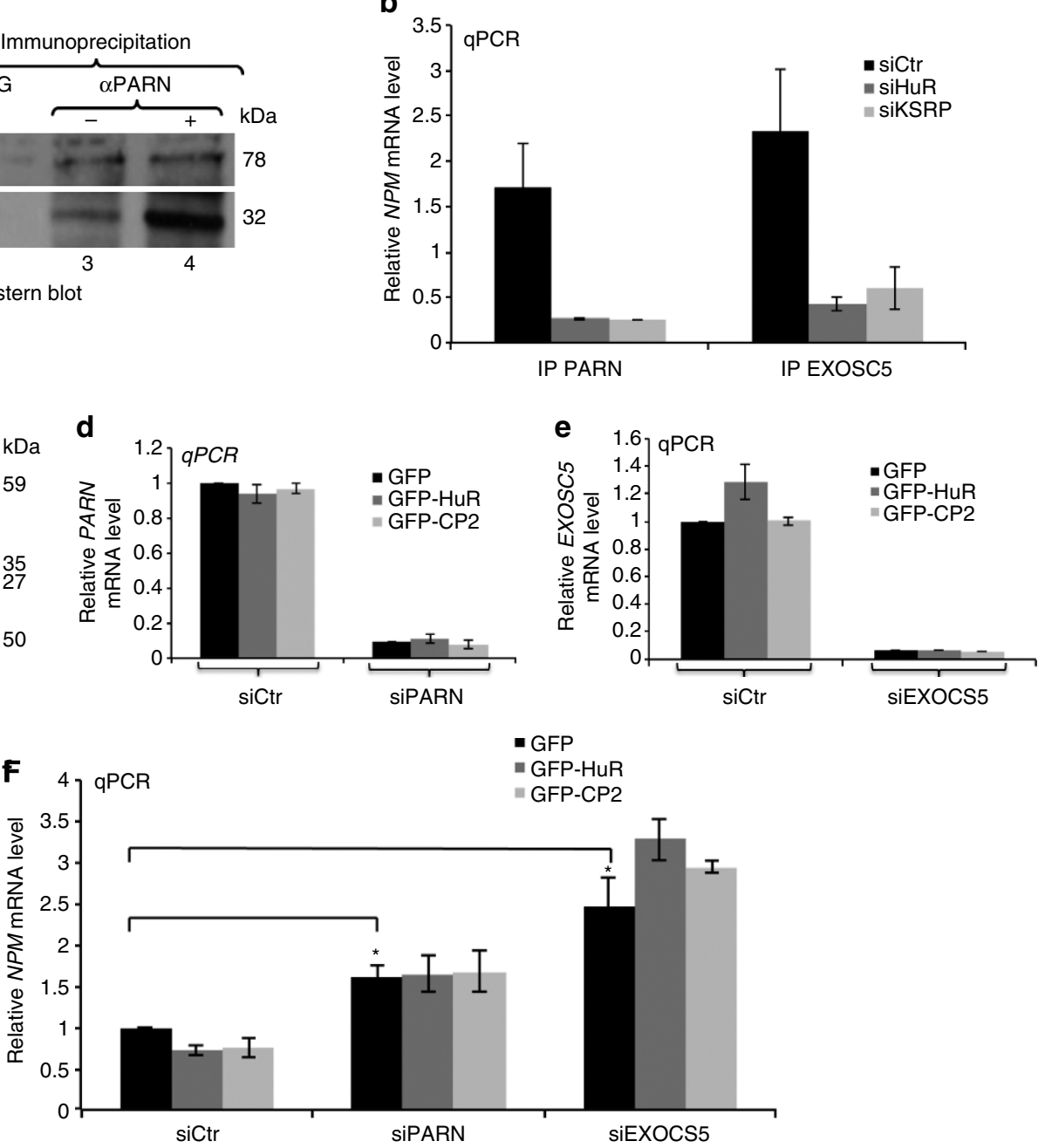

g

h

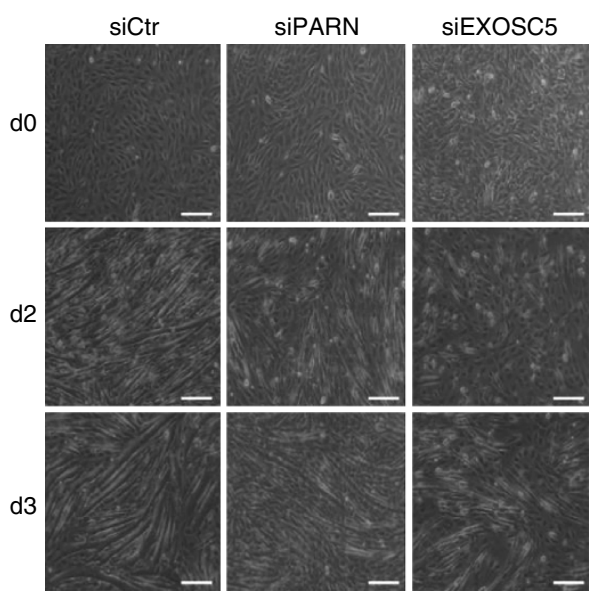

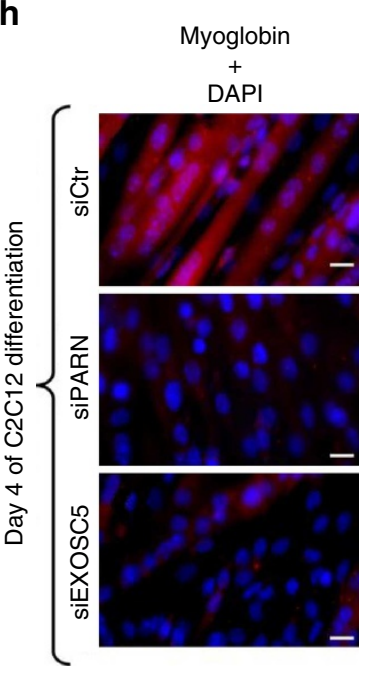

i

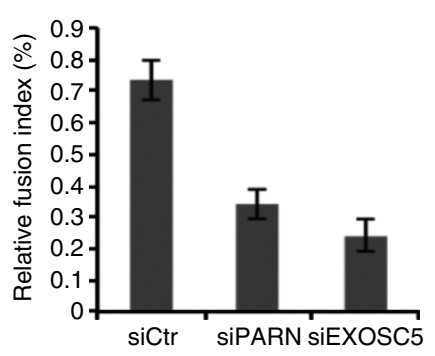

j Day 4 of $\mathrm{COC}_{12}$ differentaion

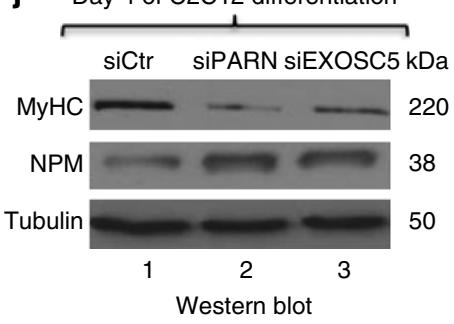


data confirmed that HuR-CP2, which harbours the RRM3 motif, but not HuR-CP1, associated with KSRP and the NPM mRNA and was also able to form a complex with the HuR/KSRP-binding element in the NPM 3'-UTR (the P1-1 probe) (Supplementary Fig. 11b-g). In addition, similarly to GFP-RRM3 (Fig. 8c), we observed that the overexpression of HuR-CP2 promoted a significant decrease in NPM mRNA steady-state levels in a KSRP dependent manner (Supplementary Fig. 11h,i). Together, these results strongly indicate that the RRM3 motif of HuR plays a key role in mediating the formation of the $\mathrm{HuR} / \mathrm{KSRP}$ in differentiating muscle cells.

Since KSRP has been shown to promote mRNA decay by recruiting the ribonuclease PARN and components of the exosome such as EXOSC5 (refs 3,17), we investigated whether enzymes could play a role in the HuR/KSRP-mediated destabilization of NPM mRNA. Our IP experiments showed that $\mathrm{HuR}$ associates with both PARN and EXOSC5 in an RNAindependent manner (Fig. 9a and Supplementary Fig. 12). Interestingly, although both PARN and EXOSC5 interact with the NPM mRNA in C2C12 cells, this association is lost in HuRor KSRP-depleted cells (Fig. 9b). To assess whether PARN and/or EXOSC5 play a role in the HuR/KSRP-mediated effect on NPM expression, we expressed GFP-HuR or GFP-HuR-CP2 in C2C12 cells depleted or not of these two ribonucleases (Fig. 9c), and the expression level of the NPM mRNA was determined using RTqPCR. Our data showed that, while knocking down PARN (Fig. 9d) or EXOSC5 (Fig. 9e) in $\mathrm{C} 2 \mathrm{C} 12$ cells increased the expression of the NPM mRNA, the absence of these ribonucleases prevented the HuR- or HuR-CP2-mediated NPM mRNA decay (Fig. 9f). The fact that the depletion of XRN1 (a $5^{\prime}-3^{\prime}$ exoribonuclease component of the decapping complex $)^{27}$ did not have a major effect on NPM mRNA expression (Supplementary Fig. 13) suggests that only ribonucleases that are recruited by KSRP such as PARN and EXOSC5 take part in this destabilization activity. Interestingly, we also demonstrate that knocking down PARN and EXOSC5 totally prevented $\mathrm{C} 2 \mathrm{C} 12$ cells from entering the differentiation process (Fig. $9 \mathrm{~g}-\mathrm{i}$ ) and the depletion of NPM in these cells partially re-established their myogenic potential (Supplementary Fig. 14). Of note, the weak rescue of myogenesis in cells depleted of both EXOSC5 and NPM suggests that EXOSC5 targets other messages in muscle cells. Together, our data indicate that during early myogenesis the formation of the HuR/KSRP complex leads to the recruitment of ribonucleases such as PARN and EXOSC5, which in turn destabilize the NPM mRNA, thus promoting muscle fibre formation.

\section{Discussion}

In this work, we delineate the molecular mechanisms by which muscle cells downregulate NPM expression and show that this event represents a key regulatory step required for their commitment to myogenesis. Although previous studies have indicated that lowering the expression of NPM is required for the differentiation of a variety of cells such as HL60 and K562 (refs $19,20)$, the molecular mechanism and the players behind this reduction remained elusive. Here we show that the decrease in NPM expression involves the destabilization of its mRNA via a mechanism mediated by the RNA-binding protein HuR. This observation was unexpected since HuR has been previously shown to stabilize the NPM mRNA in intestinal cells undergoing stress $^{18}$. In undifferentiated muscle cells, the depletion of endogenous HuR results in the stabilization of the NPM mRNA and its increased expression, while HuR overexpression elicits the opposite effects. This mRNA destabilization activity of $\mathrm{HuR}$ involves its association, via the HuR-RRM3 motif, with KSRP and the ribonucleases PARN and EXOSC5 (ref. 17). Together, our data support a model whereby in response to a myogenic signal, HuR forms a complex with KSRP, PARN and the exosome, which in turn is recruited to a U-rich element in the $3^{\prime}$-UTR, leading to the destabilization of the NPM mRNA and the promotion of the early steps of myogenesis.

Although a NPM overexpression was previously linked to the inhibition of the differentiation of human promyelocytic leukaemia HL60 cells and megakaryocytic K562 cells ${ }^{19,20}$, our work provides the first demonstration that NPM is implicated in muscle fibre formation. NPM is highly expressed in a variety of cell lines and tissues and has been involved in many cellular processes such as cell proliferation and stress response ${ }^{28,29}$. In fact, manipulating the abundance of NPM has a dramatic effect on cell fate and on embryogenesis. $\mathrm{Npm}-/-$ mice do not complete their embryonic development and die between embryonic days E11.5 and E16.5 due to a severe anaemia caused by a defect in primitive hematopoiesis ${ }^{30}$. On the other hand, a high expression level of NPM is involved in cell transformation and tumour progression ${ }^{29,31-33}$. NPM levels significantly decrease in response to a variety of stimuli, among them those known to trigger cell cycle arrest and cell differentiation ${ }^{19,20,29}$. These data and the fact that NPM and HuR are both essential for the development of numerous cell lines and tissues $12,28,29,34-36$ highlight the importance of delineating the mechanism(s) by which HuR downregulates NPM expression.

Previously, others and we have demonstrated that HuR plays a prominent role during muscle cell differentiation by increasing the stability of promyogenic mRNAs at the later stages of this process $^{5,7}$. Here we show that HuR is also involved in the early stages of myogenesis by degrading the NPM mRNA in a KSRPdependent manner. Since previous reports have indicated that during myogenesis HuR and KSRP affect the half-lives of the same promyogenic transcripts, but in opposite ways, their

\section{Figure 9 | The HuR/KSRP-mediated decay activity requires PARN and EXOSC5 to destabilize NPM mRNA and promote muscle fibre formation.}

(a) IP experiments using the anti-PARN antibody were performed on total cell extracts prepared from proliferating C2C12 cells treated or not with RNase A. The precipitates were used for western blot analysis with anti-PARN and -HuR antibodies. The blots shown are representative of two independent experiments. (b) IP coupled to RT-qPCR experiments was performed using the PARN or the EXOSC5 antibodies on total extract from C2C12 cells treated with siHuR or siKSRP. The NPM mRNA in the immunoprecipitate was standardized and normalized as described in Fig. 7i,j. The PARN- or EXOSC5associated NPM mRNA levels in siHuR- or siKSRP-treated C2C12 cells were plotted relative to siCtr conditions \pm s.e.m. of three independent experiments, ${ }^{\star} P<0.01$ ( $t$-test). (c-f) GFP, -HuR and -CP2 proteins were expressed in C2C12 cells treated or not with siRNA-PARN or -EXOSC5. Twenty-four hours later, protein extracts and total RNA were prepared. (c) Western blot analysis was performed using antibodies against GFP and $\alpha$-tubulin (loading control). (d-f) RT-qPCR analysis was performed using primers specific for GAPDH as well as PARN (d), EXOSC5 (e) and NPM (f) mRNAs. The levels of PARN, EXOSC5 and NPM mRNAs were normalized against GAPDH mRNA level and were plotted relatively to the siRNA Ctr-treated and GFP-transfected cells \pm s.e.m. of three independent experiments, ${ }^{\star} P<0.01$ ( $t$-test). ( $\mathbf{g}$-j) Confluent $\mathrm{C} 2 \mathrm{C} 12$ cells depleted of PARN or EXOSC5 were induced for differentiation for up to 4 days. (g) Phase contrast pictures showing the differentiation status of these cells at day (d) d0 (a-c), d2 (d-f) and d3 ( $g-i)$. Scale bar, $50 \mu \mathrm{m}$. (h) These cells, on day 4 of muscle cell differentiation, were also fixed and used for IF with anti-myoglobin antibody and DAPI staining. Images of a single representative field were shown. Scale bar, $10 \mu \mathrm{m}$. (i) The fusion index was determined as described in Methods. (j) Total extracts from these cells were prepared and used for western blot analysis with antibodies against My-HC, NPM and $\alpha$-tubulin (loading control). 
collaboration to degrade a common target mRNA in undifferentiated muscle cells was unexpected. Indeed, it has been shown that KSRP plays a key role in maintaining low levels of $p 21$ and myogenin mRNAs in undifferentiated muscle cells by promoting their rapid decay ${ }^{3}$. The fact that $\mathrm{HuR}$ does not associate with these mRNAs in these cells ${ }^{5}$ could explain why these transcripts became available to KSRP and the KSRPassociated decay machinery. However, during the step where muscle cells fuse to form myotubes, $\mathrm{p} 38^{\mathrm{MAPK}}$ phosphorylates KSRP leading to the release of $p 21$ and myogenin mRNAs from this decay machinery ${ }^{3}$. These findings, together with the data outlined in this manuscript, confirm the importance of HuR during muscle fibre formation and indicate that the functional consequences and the binding specificity of $\mathrm{HuR}$ to its target mRNA could change from one myogenic step to another depending on the nature of its protein ligand.

It is not surprising that HuR exerts different/opposite functions on its target transcripts. Numerous reports have indicated that depending on extra- or intracellular signals and/or the trans- or the cis-binding partners, HuR either activates or represses the turnover or translation of its mRNA targets ${ }^{10,16,37,38}$. Indeed, the Steitz laboratory has shown that HuR mediates the decay of the viral small nuclear RNA Herpesvirus saimiri U RNA 1 (HSUR 1) in an ARE-dependent manner ${ }^{39,40}$. Recently, HuR has been associated with the destabilization of the p16 mRNA both in HeLa and in IDH4 cells ${ }^{38}$. HuR participates in the decay of this message by forming a complex with AUF1 and Argonaute, two well-known mRNA decay factors ${ }^{38,41-43}$. Our data uncover a novel mechanism by which HuR mediates the decay of its mRNA target leading to muscle fibre formation. This mRNA decaypromoting activity of HuR depends on its association with KSRP, a factor known to promote the decay of ARE-containing mRNAs ${ }^{17,44}$. Interestingly, besides a difference in the way they form, the HuR/AUF1 and HuR/KSRP complexes also show a difference in their functional outcome. While in muscle cells $\mathrm{HuR} / \mathrm{KSRP}$ assemble in a RNA-independent manner before their recruitment to the same U-rich element in the NPM $3^{\prime}$-UTR, the formation of the HuR/AUF1 complex in HeLa cells is RNA dependent and occurs via the binding of each one of these two proteins with a different element in the $p 163^{\prime}-\mathrm{UTR}^{38}$. Likewise, unlike the HuR/AUF1 complex that promotes the proliferation of HeLa and IDH4 cells ${ }^{38}$, the HuR/KSRP complex triggers the differentiation of muscle cells, an event linked to a cell cycle withdrawal ${ }^{21}$.

Besides the association with different protein ligands, we still do not know whether other processes such as post-translational modifications are also involved in the functional switch of HuR during myogenesis. It has been shown that phosphorylation on various serine residues could impact the association of HuR with some of its target mRNAs such as p21 (refs 45-47). While the implication of phosphorylation in HuR-mediated effects in muscle cells is still elusive, we recently showed that caspase-mediated cleavage plays a key role in modulating the promyogenic function of HuR in refs 4 and 8. Although our data indicated that HuR-CP1 promotes the cytoplasmic accumulation of HuR during the myoblast-to-myotube fusion ${ }^{4}$, the function of HuR-CP2 remained unclear. Here we demonstrate that HuR-CP2 associates with KSRP and is sufficient to downregulate the expression of NPM mRNA in muscle cells (Supplementary Fig. 11). Interestingly, we also show that, unlike the non-cleavable isoform of HuR (HuRD226A), HuR-CP2 can re-establish the myogenic potential of $\mathrm{C} 2 \mathrm{C} 12$ cells depleted of endogenous HuR (Fig. 2i-l). While additional experiments are needed to confirm the role of this cleavage product in the promyogenic function of $\mathrm{HuR}$, these observations raise the possibility of its implication in maintaining a low expression of levels of NPM during myogenesis. While these and likely other modifications modulate HuR function in muscle cells, the data described above establish that HuR, through its ability to promote the decay of target mRNAs, acquires more flexibility to use different ways to impact important processes such as muscle fibre formation. Therefore, since a change in HuR function and associated partners has been linked to chronic and deadly diseases such as cancer and muscle wasting ${ }^{48-50}$, delineating the molecular mechanisms behind the HuR-mediated mRNA decay activity could identify novel targets that help design effective strategies to treat these patients.

\section{Methods}

Plasmid construction. The pYX-Asc plasmid containing the full-length mouse NPM cDNA (Accession Number: BC054755) was purchased from Open Biosystems (Catalogue Number: MMM1013-9497870). To generate the GFP-NPM plasmid, full-length mouse NPM was amplified by PCR using the pYX-Asc plasmid as template and the following primers: forward $5^{\prime}$-GGCAAGCTTCGTCTGTTCT GTGGAACAGGA- $3^{\prime}$ and reverse $5^{\prime}$-CGGGATCCGGGAAAGTTCTCACTTTGC ATT- $3^{\prime}$. The pAcGFP1-C1 (Clontech) and the PCR products were digested by HindIII and BamHI restriction enzymes (NE Biolabs). To generate the pRL-SV40NPM plasmid, the full-length $3^{\prime}$-UTR of mouse NPM was amplified by PCR using the pYX-Asc plasmid as template and the following primers: forward $5^{\prime}$-GCTCTA GAGAAAAGGGTTTAAACAGTTTGA- $3^{\prime}$ and reverse $5^{\prime}$-CCGGCGGCCGCAC TTTATTAAAATACTGAGTTTATT- $3^{\prime}$. The pRL-SV40 vector (Promega) and the PCR products were digested by XbaI and NotI restriction enzymes (NE Biolabs). PCR inserts were ligated into the plasmids using the T4 DNA ligase (NE Biolabs) according to the manufacturer's instructions. pRL-SV40-NPMmut1 and mut2 plasmids were generated by Norclone Biotech Laboratories, Kingstone, ON, Canada (as described in text). The GFP and GFP-HuR plasmids were generated and used as described in ref. 51.

Cell culture and transfection. C2C12 muscle cells (ATCC, Manassas, VA, USA) were grown in a media containing $20 \%$ fetal bovine serum (Invitrogen) in DMEM (Dulbecco's modified Eagle medium from Invitrogen). To induce muscle cell differentiation, cells were switched to a media containing DMEM, $2 \%$ horse serum, penicillin/streptomycin antibiotics (Invitrogen) and $50 \mathrm{mM}$ HEPES, $\mathrm{pH} 7.4$ (Invitrogen), when their confluency reached $100 \% 5,6$.

For the generation of stable cell lines, $\mathrm{C} 2 \mathrm{C} 12$ cells were transfected with GFP, GFP-HuR and GFP-NPM plasmids, and $24 \mathrm{~h}$ post-transfection G418 $\left(1 \mathrm{mg} \mathrm{ml}^{-1}\right.$ Sigma-Aldrich) was added to the media. The stable cell lines were selected with G418 for 2 weeks and then sorted by flow cytometry (fluorescence-activated cell sorting) to select clones with equal expression levels. Transfections with siRNAs specific for HuR were performed using jetPEI (Polyplus Transfection) according to the manufacturer's instructions. For DNA plasmid transfections, C2C12 cells at $70 \%$ confluency were transfected into six-well plates with $1.5 \mu \mathrm{g}$ of plasmid DNA using Lipofectamine and Plus reagents (Invitrogen) according to the manufacturer's instructions. Cells were subsequently incubated at $37^{\circ} \mathrm{C}$ for $24 \mathrm{~h}$ before collecting and analysis.

siRNA. siRNA oligonucleotides against HuR (5'-AAGCCUGUUCAGCAGCAUU GG-3' (ref. 5)), NPM (5'-CAUCAACACCGAGAUCAAAdTdT-3') (Dharmacon, USA), KSRP ( $5^{\prime}$-GGACAGUUUCACGACAACGdTdT-3' (ref. 3)) and the siRNA Ctr $\left(5^{\prime}\right.$-AAGCCAAUUCAUCAGCAAUGG- $\left.3^{\prime}\right)$ ref. 5 were synthesized by Dharmacon, USA. siRNA-PARN (5'-GGAUGUCAUGCAUACGAUUtt- $3^{\prime}$ ), EXOSC5 (5'-UCUUCAAGGUGAUACCUCUtt- $\left.3^{\prime}\right)$ and XRN1 (5'-GAGGUGU UGUUUCGAAUUAtt- $3^{\prime}$ ) were acquired from Ambion, USA.

Preparation of cell extracts and immunoblotting. Total cell extracts were prepared $^{52}$ and western blot was performed using antibodies against $\mathrm{HuR}$ (3A2, $1: 10,000)^{53}$, NPM (anti-B23 clone FC82291, Sigma-Aldrich, 1:30,000), My-HC (Developmental studies Hybridoma $\left.{ }^{5}, 1: 1,000\right)$, Myoglobin (DAKO, 1:500), $\alpha$-tubulin (Developmental studies Hybridoma Bank, 1:1,000), GFP (Clontech, Mountain View, CA, USA, 1:1,000), and KSRP (affinity-purified rabbit serum ${ }^{17}$, 1:3,000), anti-myogenin (F5D, obtained from Developmental Studies Hybridoma Bank, 1:250) and caspase 3 cleavage product (Cell Signaling Technology, 1:1,000). Scans of the most important blots are shown in Supplementary Fig. 15.

Immunofluorescence. Immunofluorescence ${ }^{5}$ was performed to detect GFP expression as well as to visualize myotubes using antibodies against $\mathrm{My}-\mathrm{HC}$ $(1: 1,000)$ and Myoglobin (1:500). Staining with 4',6-diamidino-2-phenylindole (DAPI) was employed to visualize nuclei. 
Fusion index. The fusion index ${ }^{5}$ indicating the efficiency of $\mathrm{C} 2 \mathrm{C} 12$ differentiation was determined by calculating the number of nuclei in each microscopic field in relation to the number of nuclei in myotubes in the same field.

Northern blot analysis and actinomycin D pulse-chase experiments. The extraction of total RNA from $\mathrm{C} 2 \mathrm{C} 12$ cells was performed using Trizol reagent (Invitrogen) according to the manufacturer's instructions. Northern blot analysis ${ }^{23}$ was performed using probes specific for MyoD, HuR, $18 S^{48}$ and NPM (Supplementary Table 2). These probe RNAs were generated using the PCR Purification Kit (GE Healthcare) and radiolabelled with $\alpha^{32} \mathrm{P}$ deoxycytidine triphosphate (dCTP) using Ready-to-Go DNA labelling beads (GE Healthcare) according to the manufacturer's instructions. The stability of NPM mRNA was assessed by the addition of the transcriptional inhibitor actinomycin D $\left(5 \mu \mathrm{g} \mathrm{ml}^{-1}\right.$; Sigma-Aldrich $)^{48}$ for the indicated periods of time.

RT-qPCR. $1 \mu \mathrm{g}$ of total RNA was reverse transcribed using the M-MuLV RT system. A 1/80 dilution of cDNA was used to detect the mRNAs using SsoFast EvaGreen Supermix (Bio-Rad). Expression of NPM, MyoD, $p 21$, myogenin and $R L u c$ was standardized using GAPDH as a reference (see primers in Supplementary Table 2), and relative levels of expression were quantified by calculating $2-\Delta \Delta C_{\mathrm{T}}$, where $\Delta \Delta C_{\mathrm{T}}$ is the difference in $C_{\mathrm{T}}$ (cycle number at which the amount of amplified target reaches a fixed threshold) between target and reference.

Genomic DNA extracts and analysis by RT-qPCR. Cell pellets are resuspended in the digestion buffer $(100 \mathrm{mM} \mathrm{NaCl} ; 10 \mathrm{mM}$ Tris- $\mathrm{HCl}, \mathrm{pH} 8 ; 25 \mathrm{mM}$ EDTA, $\mathrm{pH} 8 ; 0.5 \% \mathrm{SDS} ; 0.1 \mathrm{mg} \mathrm{ml}^{-1}$ proteinase $\left.\mathrm{K}\right)$ and incubated at $50^{\circ} \mathrm{C}$ overnight. Genomic DNA (gDNA) is extracted with phenol chloroform (Invitrogen) according to the manufacturer's instructions. RT-qPCR experiment was performed as described above starting from $16 \mathrm{pg}$ of extracted gDNA.

Immunoprecipitation. Cell extracts were prepared in the lysis buffer (50 mM Tris, $\mathrm{pH} 8 ; 0.5 \%$ Triton X-100; $150 \mathrm{mM} \mathrm{NaCl}$; complete protease inhibitor (Roche)). When indicated, cell extracts were digested for $30 \mathrm{~min}$ at $37^{\circ} \mathrm{C}$ with RNase A $\left(100 \mu \mathrm{g} \mathrm{ml}^{-1}\right)$. Anti-KSRP serum $(2 \mu \mathrm{l})$ (affinity-purified rabbit serum ${ }^{17}$ ) was incubated with $50 \mu$ l of proteinA-sepharose slurry beads (washed and equilibrated in cell lysis buffer) for $1 \mathrm{~h}$ at $4{ }^{\circ} \mathrm{C}$. Beads were washed three times with cell lysis buffer and incubated with $300 \mu \mathrm{g}$ of cell extracts overnight at $4^{\circ} \mathrm{C}$. Beads were washed three times with cell lysis buffer and co-immunoprecipitated proteins were analysed by western blot. HuR, PARN and EXOSC5 IP were performed ${ }^{3,5,54}$ using antibodies against HuR (3A2), PARN (Cell Signaling, USA), EXOSC5 (Abcam, USA) and IgG (Jackson Immunoresearch Laboratories, USA).

Preparation of mRNA (mRNP) complexes and analysis by RT-PCR. Purified RNA from mRNP complexes ${ }^{5,54}$ was resuspended in $10 \mu \mathrm{l}$ of water and $4 \mu \mathrm{l}$ was reverse transcribed using the M-MuLV RT system (NE Biolabs) according to the manufacturer's protocol. Subsequently, $2 \mu \mathrm{l}$ of cDNA was amplified by PCR or qPCR using NPM-specific primers (Supplementary Table 2). When analysed by qPCR, a 1/20 dilution of cDNA was used to detect the mRNAs using SybrGreen (SsoFast EvaGreen Supermix). The mRNA levels associated with these mRNP complexes were then standardized against RPL32 mRNA levels (used as a reference) and compared with mRNA levels in the IgG control. In experiments transfected with Rluc reporter constructs, the Rluc mRNA associated with immunoprecipiated HuR was determined by RT-qPCR, standardized against RPL32 mRNA levels and then the steady-state levels for each treatment. These standardized Rluc mRNA levels were then compared with Rluc mRNA levels in the IgG IP.

cDNA array analysis. Microarray experiments were performed using mouse array, which contain probe sets of characterized and unknown mouse expressed sequence tags (ESTs) from 17,000 genes ${ }^{55}$. RNAs from siHuR- and siCtr-treated $\mathrm{C} 2 \mathrm{C} 12$ cell extracts were prepared ${ }^{56}$, processed and hybridized on the arrays. The data were processed using the Array Pro software (Media Cybernetics), then normalized by $Z$-score transformation ${ }^{55}$ and used to calculate differences in signal intensities. Significant values were tested using a two-tailed $Z$-test and a $P$-value of $\leq 0.01$. The data were calculated from two independent experiments.

Fluorescence in situ hybridization. The fluorescence in situ hybridization experiments were performed ${ }^{57}$ using a DNA fragment of $\sim 500$ bp corresponding to the coding region of mouse NPM. The fragment was amplified by PCR using the following primers fused to either a T7 or T3 minimal promoter sequence: NPM forward, 5' -TAATACGACTCACTATAGGGACGGTTGAAGTGTGGTTCAG-3' and NPM reverse, $5^{\prime}$-AATTAACCCTCACTAAAGGAACTTGGCTTCCACTT TGG-3'. The PCR product was used as the template for in vitro transcription of the NPM probe needed for fluorescence in situ hybridization. The antisense (T3) and sense (T7) probes were prepared using digoxigenin-RNA labelling mix (Roche Diagnostics). The RNA probes were quantified, denatured and incubated with permeabilized cells ${ }^{57}$. After the hybridization, the cells were used for immunofluorescence to detect $\mathrm{HuR}^{57}$.

Polysome fractionation. A total of $4 \times 10^{7}$ myoblasts were grown and treated with siRNAs as described above. Briefly, the cytoplasmic extracts obtained from lysed myoblast cells were centrifuged at $130,000 \mathrm{~g}$ for $2 \mathrm{~h}$ on a sucrose gradient $(10-50 \%$ $\mathrm{w} / \mathrm{v})^{58}$. RNA was extracted using Trizol LS (Invitrogen), and was then analysed on an agarose gel. The levels of NPM and GAPDH mRNAs were determined by RT-qPCR.

RNA electrophoretic mobility shift assays. The NPM cRNA probes were produced by in vitro transcription ${ }^{59}$. The accession number in the NCBI database of the NPM mRNA sequence used to generate these probes is NM_008722. The NPM probes $5^{\prime}$-UTR, $3^{\prime}$-UTR, P1 and P2 were generated by PCR amplification using a forward primer fused to the T7 promoter (Supplementary Table 2) as well as pYX-Asc-NPM expression vector as the template. For smaller probes (P1-1 to P2-3, P1-1-mut1, P1-1-mut2, P1-1-mut1-2), oligonucleotide sense and antisense (see Supplementary Materials and Methods) were directly annealed and used for in vitro transcription. The RNA-binding assays ${ }^{23}$ were performed using $500 \mathrm{ng}$ purified recombinant protein (GST or GST-HuR) incubated with 50,000 c.p.m. of ${ }^{32} \mathrm{P}$-labelled cRNAs. Supershift experiments were also performed with $10 \mu \mathrm{g}$ total C2C12 cell extract incubated with 50,000 c.p.m. of ${ }^{32} \mathrm{P}$-labelled cRNAs. An anti-KSRP antibody was then added to the reaction to supershift the RNP/cRNA complex containing KSRP.

Luciferase activity. The activity of Renilla luciferase was measured using a Renilla luciferase assay system (Promega) with a luminometer following the manufacturer's instructions.

In vitro pull-down experiments. GST pull-down assay ${ }^{4}$ was performed using GST-HuR and recombinant His-KSRP. The interaction of the His-KSRP with the pulled-down GST-HuR was analysed by western blot using the GST and KSRP antibodies.

\section{References}

1. Tallquist, M. D., Weismann, K. E., Hellstrom, M. \& Soriano, P. Early myotome specification regulates PDGFA expression and axial skeleton development. Development 127, 5059-5070 (2000).

2. Jun, J. I. \& Lau, L. F. The matricellular protein CCN1 induces fibroblast senescence and restricts fibrosis in cutaneous wound healing. Nat. Cell Biol. 12, 676-685 (2010).

3. Briata, P. et al. p38-dependent phosphorylation of the mRNA decay-promoting factor ksrp controls the stability of select myogenic transcripts. Mol. Cell 20, 891-903 (2005).

4. Beauchamp, P. et al. The cleavage of HuR interferes with its transportin-2mediated nuclear import and promotes muscle fiber formation. Cell Death Differ. 17, 1588-1599 (2010).

5. van der Giessen, K., Di-Marco, S., Clair, E. \& Gallouzi, I. E. RNAi-mediated HuR depletion leads to the inhibition of muscle cell differentiation. J. Biol. Chem. 278, 47119-47128 (2003).

6. van der Giessen, K. \& Gallouzi, I. E. Involvement of transportin 2-mediated HuR import in muscle cell differentiation. Mol. Biol. Cell 18, 2619-2629 (2007).

7. Figueroa, A. et al. Role of HuR in skeletal myogenesis through coordinate regulation of muscle differentiation genes. Mol. Cell. Biol. 23, 4991-5004 (2003).

8. von Roretz, C., Beauchamp, P., Di Marco, S. \& Gallouzi, I. E. HuR and myogenesis: being in the right place at the right time. Biochim. Biophys. Acta 1813, 1663-1667 2011.

9. von Roretz, C. \& Gallouzi, I. E. Decoding ARE-mediated decay: is microRNA part of the equation? J. Cell Biol. 181, 189-194 (2008).

10. Abdelmohsen, K., Kuwano, Y., Kim, H. H. \& Gorospe, M. Posttranscriptional gene regulation by RNA-binding proteins during oxidative stress: implications for cellular senescence. Biol. Chem. 389, 243-255 (2008).

11. Lopez de Silanes, I., Zhan, M., Lal, A., Yang, X. \& Gorospe, M. Identification of a target RNA motif for RNA-binding protein HuR. Proc. Natl Acad. Sci. USA 101, 2987-2992 (2004).

12. Brennan, C. M. \& Steitz, J. A. HuR and mRNA stability. Cell. Mol. Life Sci. 58, 266-277 (2001)

13. Gallouzi, I. E., Brennan, C. M. \& Steitz, J. A. Protein ligands mediate the CRM1-dependent export of HuR in response to heat shock. RNA 7, 1348-1361 (2001).

14. Gallouzi, I. E. \& Steitz, J. A. Delineation of mRNA export pathways by the use of cell-permeable peptides. Science 294, 1895-1901 (2001).

15. Abdelmohsen, K., Srikantan, S., Kuwano, Y. \& Gorospe, M. miR-519 reduces cell proliferation by lowering RNA-binding protein HuR levels. Proc. Natl Acad. Sci. USA 105, 20297-20302 (2008). 
16. Kim, H. H. et al. HuR recruits let-7/RISC to repress c-Myc expression. Genes Dev. 23, 1743-1748 (2009).

17. Gherzi, R. et al. A KH domain RNA binding protein, KSRP, promotes ARE-directed mRNA turnover by recruiting the degradation machinery. Mol. Cell 14, 571-583 (2004).

18. Zou, T. et al. Polyamine depletion increases cytoplasmic levels of RNA-binding protein HuR leading to stabilization of nucleophosmin and p53 mRNAs. J. Biol. Chem. 281, 19387-19394 (2006).

19. Hsu, C. Y. \& Yung, B. Y. Over-expression of nucleophosmin/B23 decreases the susceptibility of human leukemia HL-60 cells to retinoic acid-induced differentiation and apoptosis. Int. J. Cancer 88, 392-400 (2000).

20. Hsu, C. Y. \& Yung, B. Y. Involvement of nucleophosmin/B23 in TPA-induced megakaryocytic differentiation of K562 cells. Br. J. Cancer 89, 1320-1326 (2003).

21. Charge, S. B. \& Rudnicki, M. A. Cellular and molecular regulation of muscle regeneration. Physiol. Rev. 84, 209-238 (2004).

22. Garcia-Martinez, J., Aranda, A. \& Perez-Ortin, J. E. Genomic run-on evaluates transcription rates for all yeast genes and identifies gene regulatory mechanisms. Mol. Cell 15, 303-313 (2004).

23. Dormoy-Raclet, V. et al. The RNA-binding protein HuR promotes cell migration and cell invasion by stabilizing the beta-actin mRNA in a U-rich-element-dependent manner. Mol. Cell. Biol. 27, 5365-5380 (2007).

24. Barreau, C., Paillard, L. \& Osborne, H. B. AU-rich elements and associated factors: are there unifying principles? Nucleic Acids Res. 33, $7138-7150$ (2005)

25. Sanduja, S., Kaza, V. \& Dixon, D. A. The mRNA decay factor tristetraprolin (TTP) induces senescence in human papillomavirus-transformed cervical cancer cells by targeting E6-AP ubiquitin ligase. Aging (Albany NY) 1, 803-817 (2009).

26. Drury, G. L., Di Marco, S., Dormoy-Raclet, V., Desbarats, J. \& Gallouzi, I. E. FasL expression in activated T lymphocytes involves HuR-mediated stabilization. J. Biol. Chem. 285, 31130-31138 (2010).

27. Long, R. M. \& McNally, M. T. mRNA decay: x (XRN1) marks the spot. Mol. Cell 11, 1126-1128 (2003)

28. Frehlick, L. J., Eirin-Lopez, J. M. \& Ausio, J. New insights into the nucleophosmin/nucleoplasmin family of nuclear chaperones. Bioessays 29, 49-59 (2007).

29. Grisendi, S., Mecucci, C., Falini, B. \& Pandolfi, P. P. Nucleophosmin and cancer. Nat. Rev. Cancer 6, 493-505 (2006).

30. Grisendi, S. et al. Role of nucleophosmin in embryonic development and tumorigenesis. Nature 437, 147-153 (2005).

31. Gubin, A. N., Njoroge, J. M., Bouffard, G. G. \& Miller, J. L. Gene expression in proliferating human erythroid cells. Genomics 59, 168-177 (1999).

32. Feuerstein, N. \& Randazzo, P. A. In vivo and in vitro phosphorylation studies of numatrin, a cell cycle regulated nuclear protein, in insulin-stimulated NIH 3T3 HIR cells. Exp. Cell Res. 194, 289-296 (1991).

33. Dergunova, N. N. et al. A major nucleolar protein B23 as a marker of proliferation activity of human peripheral lymphocytes. Immunol. Lett. 83, 67-72 (2002).

34. Katsanou, V. et al. The RNA-binding protein Elavl1/HuR is essential for placental branching morphogenesis and embryonic development. Mol. Cell. Biol. 29, 2762-2776 (2009).

35. Katsanou, V. et al. HuR as a negative posttranscriptional modulator in inflammation. Mol. Cell 19, 777-789 (2005).

36. Papadaki, O. et al. Control of thymic T cell maturation, deletion and egress by the RNA-binding protein HuR. J. Immunol. 182, 6779-6788 (2009).

37. Bhattacharyya, S. N., Habermacher, R., Martine, U., Closs, E. I. \& Filipowicz, W. Relief of microRNA-mediated translational repression in human cells subjected to stress. Cell 125, 1111-1124 (2006).

38. Chang, N. et al. HuR uses AUF1 as a cofactor to promote pl6INK4 mRNA decay. Mol. Cell. Biol. 30, 3875-3886 (2010).

39. Fan, X. C., Myer, V. E. \& Steitz, J. A. AU-rich elements target small nuclear RNAs as well as mRNAs for rapid degradation. Genes Dev. 11, 2557-2568 (1997).

40. Myer, V. E., Fan, X. C. \& Steitz, J. A. Identification of HuR as a protein implicated in AUUUA-mediated mRNA decay. EMBO J. 16, 2130-2139 (1997).

41. DeMaria, C. T. \& Brewer, G. AUF1 binding affinity to A + U-rich elements correlates with rapid mRNA degradation. J. Biol. Chem. 271, 12179-12184 (1996).

42. Thomas, M., Lieberman, J. \& Lal, A. Desperately seeking microRNA targets. Nat. Struct. Mol. Biol. 17, 1169-1174 (2011).

43. Parker, R. \& Sheth, U. P bodies and the control of mRNA translation and degradation. Mol. Cell 25, 635-646 (2007).

44. Gherzi, R. et al. The RNA-binding protein KSRP promotes decay of beta-catenin mRNA and is inactivated by PI3K-AKT signaling. PLoS Biol. 5, e5 (2006).

45. Masuda, K. et al. Global dissociation of HuR-mRNA complexes promotes cell survival after ionizing radiation. EMBO J. 30, 1040-1053 (2011).
46. Abdelmohsen, K., Lal, A., Kim, H. H. \& Gorospe, M. Posttranscriptional orchestration of an anti-apoptotic program by HuR. Cell Cycle 6, 1288-1292 (2007).

47. Lafarga, V. et al. p38 Mitogen-activated protein kinase- and HuR-dependent stabilization of p21(Cip1) mRNA mediates the G(1)/S checkpoint. Mol. Cell. Biol. 29, 4341-4351 (2009).

48. Di Marco, S. et al. NF-(kappa)B-mediated MyoD decay during muscle wasting requires nitric oxide synthase mRNA stabilization, HuR protein, and nitric oxide release. Mol. Cell. Biol. 25, 6533-6545 (2005).

49. Lopez de Silanes, I. et al. Global analysis of HuR-regulated gene expression in colon cancer systems of reducing complexity. Gene Expr. 12, 49-59 (2004).

50. Lopez de Silanes, I., Lal, A. \& Gorospe, M. HuR: post-transcriptional paths to malignancy. RNA Biol. 2, 11-13 (2005).

51. Mazroui, R. et al. Caspase-mediated cleavage of HuR in the cytoplasm contributes to pp32/PHAP-I regulation of apoptosis. J. Cell Biol. 180, 113-127 (2008).

52. von Roretz, C. \& Gallouzi, I. E. Protein kinase RNA/FADD/caspase-8 pathway mediates the proapoptotic activity of the RNA-binding protein human antigen R (HuR). J. Biol. Chem. 285, 16806-16813 (2010).

53. Gallouzi, I. E. et al. HuR binding to cytoplasmic mRNA is perturbed by heat shock. Proc. Natl Acad. Sci. USA 97, 3073-3078 (2000).

54. Tenenbaum, S. A., Lager, P. J., Carson, C. C. \& Keene, J. D. Ribonomics: identifying mRNA subsets in mRNP complexes using antibodies to RNA binding proteins and genomic arrays. Methods 26, 191-198 (2002).

55. Cheadle, C., Vawter, M. P., Freed, W. J. \& Becker, K. G. Analysis of microarray data using Z score transformation. J. Mol. Diagn. 5, 73-81 (2003).

56. Lopez de Silanes, I. et al. Identification and functional outcome of mRNAs associated with RNA-binding protein TIA-1. Mol. Cell. Biol. 25, 9520-9531 (2005).

57. Lian, X. J. \& Gallouzi, I. E. Oxidative stress increases the number of stress granules in senescent cells and triggers a rapid decrease in p21waf1/cip1 translation. J. Biol. Chem. 284, 8877-8887 (2009).

58. Cammas, A. et al. Cytoplasmic relocalization of heterogeneous nuclear ribonucleoprotein A1 controls translation initiation of specific mRNAs. Mol. Biol. Cell 18, 5048-5059 (2007).

59. Gallouzi, I. E. et al. A novel phosphorylation-dependent RNase activity of GAP-SH3 binding protein: a potential link between signal transduction and RNA stability. Mol. Cell. Biol. 18, 3956-3965 (1998).

\section{Acknowledgements}

We thank Dr C. von Roretz for critical review of this manuscript and insightful discussion. We are grateful to Veronique Sauve and Dr Kalle Gehring for their help with the BIACORE experiments. A.C. is a recipient of the Canderal scholarship for PDFs (2010-2011) as well as the Peter Quinlan Fellowship in Oncolgy (2011-2012). V.D.-R. is a Research Fellow of the Terry Fox foundation through an award from the Canadian Cancer Society (former National Cancer Institute of Canada). This work was supported by a CIHR (MOP-89798) operating grant to I.-E.G. M.G. is supported by the NIA-IRP, NIH. R.G. is supported by grants from AIRC (IG 2010-2012) and AICR (No. 10-0527) I.-E.G. is a recipient of a TierII Canada Research Chair.

\section{Author contributions}

A.C. designed, carried out, analysed and interpreted all of the experiments in the manuscript. B.J.S., V.D.-R., X.J.L., K.v.d.G. and J.M. assisted with some of the immuno/ northern blots as well as RT-qPCR experiments described in the manuscript. I.L.d.S. and M.G. designed and carried out the microarray experiments described in Supplementary Table 1. M.G. and R.G. helped with the IP experiment described in Fig. 7f. R.G. also helped with the design of experiments described inFigs 6 and 7 and provided reagents C.W., J.R. and S.M. provided technical expertise and helped in the design of some of the experiments. S.D. helped in the design of several experiments in the manuscript. S.D. also participated in the writing and editing the manuscript. I.-E.G. conceived and helped design all experiments in the manuscript, aided in the analysis of the data and wrote the manuscript.

\section{Additional information}

Accession Codes: Microarray data has been deposited in Gene Expression Omnibus under accession number GSE57113.

Supplementary Information accompanies this paper at http://www.nature.com/ naturecommunications

Competing financial interests: The authors declare no competing financial interests.

Reprints and permission information is available online at http://npg.nature.com/ reprintsandpermissions/

How to cite this article: Cammas, A. et al. Destabilization of nucleophosmin mRNA by the HuR/KSRP complex is required for muscle fibre formation. Nat. Commun. 5:4190 doi: $10.1038 /$ ncomms5190 (2014) 